\title{
EQUIVARIANT REPRESENTABLE K-THEORY
}

\author{
HEATH EMERSON AND RALF MEYER
}

\begin{abstract}
We interpret certain equivariant Kasparov groups as equivariant representable K-theory groups and compute these via a classifying space and as $\mathrm{K}$-theory groups of suitable $\sigma-C^{*}$-algebras. We also relate equivariant vector bundles to these $\sigma$ - $C^{*}$-algebras and provide sufficient conditions for equivariant vector bundles to generate representable K-theory. We mostly work in the generality of locally compact groupoids with Haar system.
\end{abstract}

\section{INTRODUCTION}

For a locally compact space $X$, we must distinguish its $\mathrm{K}$-theory $\mathrm{K}^{*}(X)$ and its representable K-theory $\mathrm{RK}^{*}(X)$. Vector bundles on $X$ define classes in $\mathrm{RK}^{*}(X)$, but not necessarily in $\mathrm{K}^{*}(X)$. Furthermore, given a map $X \rightarrow Y$, we can define $\operatorname{RK}_{Y}^{*}(X)$, the K-theory of $X$ with $Y$-compact support. Such groups are important, for instance, because the symbol of an elliptic pseudodifferential operator on a smooth manifold $X$ lies in the group $\operatorname{RK}_{X}^{*}(T X)$.

We may define all three theories using homotopy classes of continuous maps from $X$ to the space of Fredholm operators on a Hilbert space. For $\mathrm{RK}^{0}(X)$, we allow all such maps, for $\mathrm{K}^{0}(X)$, we require the map to have unitary values outside a compact subset, and for $\operatorname{RK}_{Y}^{*}(X)$, we require unitary values outside a $Y$-compact subset of $X$.

Alternatively, we may identify $\mathrm{RK}^{*}(X)$ and $\mathrm{RK}_{Y}^{*}(X)$ with the equivariant Kasparov groups $\mathrm{KK}_{*}^{X}\left(\mathcal{C}_{0}(X), \mathcal{C}_{0}(X)\right)$ and $\mathrm{KK}_{*}^{Y}\left(\mathcal{C}_{0}(Y), \mathcal{C}_{0}(X)\right)$, respectively, where we view $X$ and $Y$ as groupoids with only identity morphisms (these groups are denoted by $\operatorname{RKK}(X ; \mathbb{C}, \mathbb{C}) \cong \mathcal{R} \operatorname{KK}\left(X ; \mathcal{C}_{0}(X), \mathcal{C}_{0}(X)\right)$ and $\mathcal{R} \operatorname{KK}\left(Y ; \mathcal{C}_{0}(Y), \mathcal{C}_{0}(X)\right)$ in [17]).

In this article, we study equivariant versions of these three kinds of K-theory. We establish an equivalence between several alternative definitions that are based on equivariant Kasparov theory, maps to spaces of Fredholm operators, K-theory of certain crossed product $\sigma$ - $C^{*}$-algebras, and equivariant vector bundles, respectively. The main goal here is to show that certain equivariant Kasparov groups deserve to be called "representable equivariant K-theory" or "equivariant K-theory with $Y$-compact support." Moreover, we study the general properties of these theories and investigate when representable equivariant K-theory can be described by equivariant vector bundles.

Although we are mainly interested in the case of group actions, we must consider crossed product groupoids $G \ltimes X$, anyway. Therefore, we work with groupoids most of the time. More precisely, we use Hausdorff, second countable, locally compact groupoids with Haar systems. Let $\mathcal{G}$ be such a groupoid.

Let $X$ be a (Hausdorff, locally compact, second countable) proper $\mathcal{G}$-space, that is, the crossed product groupoid $\mathcal{G} \ltimes X$ is proper. We define the $\mathcal{G}$-equivariant K-theory of $X$ by

$$
\mathrm{K}_{\mathcal{G}}^{*}(X):=\mathrm{K}_{*}\left(\mathcal{G} \ltimes \mathcal{C}_{0}(X)\right) .
$$

If $\mathcal{G}$ is a group, this agrees with Chris Phillips' definition in [28].

2000 Mathematics Subject Classification. 19K35, 46L80. 
The $\mathcal{G}$-equivariant representable $\mathrm{K}$-theory of $X$ is defined by

$$
\operatorname{RK}_{\mathcal{G}}^{*}(X):=\mathrm{KK}_{*}^{\mathcal{G} \ltimes X}\left(\mathcal{C}_{0}(X), \mathcal{C}_{0}(X)\right) .
$$

Let $Y$ be another $\mathcal{G} \ltimes X$-space. The $\mathcal{G}$-equivariant K-theory of $Y$ with $X$-compact supports is defined by

$$
\operatorname{RK}_{\mathcal{G}, X}^{*}(Y):=\mathrm{KK}_{*}^{\mathcal{G} \ltimes X}\left(\mathcal{C}_{0}(X), \mathcal{C}_{0}(Y)\right) .
$$

Of course, $\mathrm{RK}_{\mathcal{G}}^{*}(X)$ is the special case $\operatorname{RK}_{\mathcal{G}, X}^{*}(X)$ of the last definition. Conversely, $\operatorname{RK}_{\mathcal{G}, X}^{*}(Y)$ can be computed as the inductive limit of the groups $\operatorname{RK}_{\mathcal{G}}^{*}(A, \partial A)$, where $A$ runs through the directed set of $X$-compact $\mathcal{G}$-invariant subsets of $Y$ and $\operatorname{RK}_{\mathcal{G}}^{*}(A, \partial A)$ denotes the relative version of $\mathrm{RK}_{\mathcal{G}}^{*}$ (see Theorem 4.19).

A first alternative definition of $\operatorname{RK}_{\mathcal{G}}^{*}(X)$ extends Graeme Segal's description of representable K-theory as the set of homotopy classes of maps to the space of Fredholm operators on a separable Hilbert space (33). We use the direct sum of countably many copies of the regular representation, $\mathcal{H}_{\mathcal{G}}:=\bigoplus_{n \in \mathbb{N}} L^{2}(\mathcal{G})$. This is a continuous field of Hilbert spaces over the object space $\mathcal{G}^{(0)}$ of the groupoid. Let $\mathcal{F}_{\mathcal{G}}$ be the set over $\mathcal{G}^{(0)}$ of essentially unitary operators on the corresponding fibres of $\mathcal{H}_{\mathcal{G}}$. The groupoid $\mathcal{G}$ acts on $\mathcal{F}_{\mathcal{G}}$ in an obvious way. Almost by definition, the Kasparov group $\operatorname{RK}_{\mathcal{G}}^{0}(X)$ is the space of homotopy classes of $\mathcal{G}$-equivariant maps $X \rightarrow \mathcal{F}_{\mathcal{G}}$ that are continuous in a suitable sense. But we must be careful about the topology here.

In the groupoid case, we cannot expect the continuous field of Hilbert spaces $\mathcal{H}_{\mathcal{G}}$ to be locally trivial. As a result, there is no canonical norm topology on $\mathcal{F}_{\mathcal{G}}$; only on compact operators is the norm topology canonically defined. We equip $\mathcal{F}_{\mathcal{G}}$ with the topology where a net $\left(U_{i}\right)$ converges to $U$ if and only if $U_{i} \rightarrow U$ and $U_{i}^{*} \rightarrow U^{*}$ strongly and $1-U_{i} U_{i}^{*} \rightarrow 1-U U^{*}$ and $1-U_{i}^{*} U_{i} \rightarrow 1-U^{*} U$ in norm. For this topology, $\operatorname{RK}_{\mathcal{G}}^{0}(X)$ is the space of homotopy classes of $\mathcal{G}$-equivariant continuous $\operatorname{maps} X \rightarrow \mathcal{F}_{\mathcal{G}}$.

There are similar descriptions for $\operatorname{RK}_{\mathcal{G}, Y}^{0}(X)$ and $\mathrm{K}_{\mathcal{G}}^{0}(Y)$. The support of a continuous $\mathcal{G}$-equivariant map $U: X \rightarrow \mathcal{F}_{\mathcal{G}}$ is the closure of the set of $x \in X$ where $U_{x}$ is not unitary. We identify $\operatorname{RK}_{\mathcal{G}, Y}^{0}(X)$ and $\mathrm{K}_{\mathcal{G}}^{0}(Y)$ with the sets of homotopy classes of continuous $\mathcal{G}$-equivariant maps $X \rightarrow \mathcal{F}_{\mathcal{G}}$ with $Y$-compact or $\mathcal{G}$-compact support, respectively.

For a locally compact group $G$, there is a canonical norm topology on $\mathcal{F}_{G}$. We show that it makes no difference whether we use this norm topology instead of the weaker topology described above. This requires, among other things, an equivariant version of the Cuntz-Higson-Kuiper Theorem on the contractibility of the unitary group of stable multiplier algebras of $\sigma$-unital $C^{*}$-algebras (see [8]).

We also describe $\operatorname{RK}_{\mathcal{G}, X}^{*}(Y)$ as the K-theory of a certain $\sigma$ - $C^{*}$-algebra $([26,29])$, that is, a countable inverse limit of $C^{*}$-algebras. Let $\left(X_{n}\right)_{n \in \mathbb{N}}$ be an increasing sequence of $\mathcal{G}$-compact $\mathcal{G}$-invariant subsets of $X$ with $\bigcup X_{n}=X$, and let $Y_{n}$ be the pre-image of $X_{n}$ with respect to the canonical map $Y \rightarrow X$. Then

$$
\operatorname{RK}_{\mathcal{G}, X}^{*}(Y) \cong \mathrm{K}_{*}\left(\lim _{\longleftarrow} C^{*}\left(\mathcal{G} \ltimes Y_{n}\right)\right) .
$$

Here we use Phillips' representable K-theory for $\sigma$ - $C^{*}$-algebras, so that our $\mathrm{K}_{0}$ does not agree with algebraic $\mathrm{K}_{0}$. Using the exactness properties of $\sigma$ - $C^{*}$-algebra K-theory, we define $\operatorname{RK}_{\mathcal{G}}^{*}(X, A)$ for pairs and establish excision and long exact sequences for this theory. Furthermore, we get a Milnor $\lim ^{1}$-sequence that relates $\operatorname{RK}_{\mathcal{G}, X}^{*}(Y)$ to the projective system $\left(\mathrm{K}_{\mathcal{G}}^{*}\left(Y_{n}\right)\right)_{n \in \mathbb{N}}$. Other properties like homotopy invariance and induction isomorphisms are immediate from the definitions as well.

As in 19, we also consider a version of K-theory defined by equivariant vector bundles. Let $\operatorname{VK}_{\mathcal{G}}^{0}(X)$ be the Grothendieck group of the monoid of $\mathcal{G}$-equivariant 
vector bundles on $X$. We extend this to a $\mathbb{Z} / 2$-graded theory for pairs of spaces. There is always a canonical map

$$
\operatorname{VK}_{\mathcal{G}}^{*}(X, A) \rightarrow \operatorname{RK}_{\mathcal{G}}^{*}(X, A),
$$

but it need not be an isomorphism in general, as various counterexamples in [19, 27, 32 show. We provide a sufficient condition for an isomorphism, but it is not particularly striking. Similar results about twisted equivariant K-theory for Lie groupoids are established in 35.

Our approach is to relate equivariant vector bundles to the same $\sigma$ - $C^{*}$-algebra

$$
\sigma-C^{*}(\mathcal{G} \ltimes X):=\lim _{\longleftarrow} C^{*}\left(\mathcal{G} \ltimes X_{n}\right)
$$

that already computes $\operatorname{RK}_{\mathcal{G}}^{*}(X)$. We show that $\mathcal{G}$-equivariant vector bundles on $X$ correspond to projections in the stabilisation

$$
\sigma-C^{*}(\mathcal{G} \ltimes X)_{\mathbb{K}}:=\lim _{\longleftarrow} C^{*}\left(\mathcal{G} \ltimes X_{n}\right) \otimes \mathbb{K}\left(\ell^{2} \mathbb{N}\right) .
$$

Hence the question is whether $\mathrm{K}_{0}\left(\sigma-C^{*}(\mathcal{G} \ltimes X)\right)$ is the Grothendieck group of the monoid of projections in $\sigma-C^{*}(\mathcal{G} \ltimes X)_{\mathbb{K}}$. This happens if $\sigma-C^{*}(\mathcal{G} \ltimes X)_{\mathbb{K}}$ is a $C^{*}$-algebra with an approximate unit of projections. Notice that $\sigma$ - $C^{*}(\mathcal{G} \ltimes X)_{\mathbb{K}}$ is a $C^{*}$-algebra if and only if $G$ acts cocompactly on $X$. Our positive results are limited to cocompact actions for this reason.

We show that $\sigma-C^{*}(\mathcal{G} \ltimes X)_{\mathbb{K}}$ has an approximate unit of projections if and only if for each $x \in X$ and each irreducible representation of the stabiliser of $x$, there is a $\mathcal{G}$-equivariant vector bundle on $X$ whose restriction to $x$ contains the given representation of the stabiliser. If, in addition, the action of $\mathcal{G}$ on $X$ is cocompact as well, then the map in (1.1) is an isomorphism for all closed $\mathcal{G}$-invariant subsets $A \subseteq X$ and all $* \in \mathbb{Z}$.

This sufficient criterion covers all cases where we know (1.1) to be an isomorphism: cocompact actions of almost connected groups, discrete groups, or inverse limits of discrete groups (see [19, 27, 32]), and some simple cases like equivalence relations, orbifold groupoids, and group actions that are induced from compact subgroups. A counterexample by Juliane Sauer yields examples of a totally disconnected group or of a bundle of totally disconnected compact groups over the circle for which $\operatorname{VK}_{\mathcal{G}}^{0}(X) \nRightarrow \mathrm{RK}_{\mathcal{G}}^{0}(X)$ for some space $X$.

Since any proper group action is locally induced from a compact subgroup, the problem for cocompact group actions is a purely global one. The counterexamples in [19, 28, 32 and the example of continuous trace $C^{*}$-algebras show that the existence of an approximate unit of projections in the stabilisation is a rather subtle property.

We finish the introduction with an application that motivated us to write this article. Let $X$ and $Y$ be two smooth manifolds. Then a family of elliptic pseudodifferential operators on $X$ parametrised by $Y$, on the one hand, provides an element in $\mathrm{KK}_{*}\left(\mathcal{C}_{0}(X), \mathcal{C}_{0}(Y)\right)$ and, on the other hand, has a symbol in the K-theory of $T X \times Y$ with $X$-compact support, where $T X$ denotes the tangent space of $X$. This remains true equivariantly. Let $G$ be a locally compact group, let $Y$ be a $G$-space, and let $X$ be a proper, smooth $G$-manifold with tangent bundle $T X$. The second Poincaré duality isomorphism in [13] provides a natural isomorphism

$$
\mathrm{KK}_{*}^{G}\left(\mathcal{C}_{0}(X), \mathcal{C}_{0}(Y)\right) \cong \mathrm{RK}_{G, X}^{*}(T X \times Y) .
$$

The proof of this isomorphism in [13] depends on our definition of $\operatorname{RK}_{G, X}^{*}(T X \times$ $Y)$ as an equivariant Kasparov group. It provides more geometric descriptions of $\mathrm{KK}_{*}^{G}\left(\mathcal{C}_{0}(X), \mathcal{C}_{0}(Y)\right)$ when we combine it with our alternative descriptions of $\operatorname{RK}_{G, X}^{*}(T X \times Y)$ in terms of equivariant maps to a space of Fredholm operators or, in nice cases, equivariant vector bundles. 


\section{BASIC DEFINITIONS}

Throughout this article, we act as if we were dealing with complex $C^{*}$-algebras and complex K-theory. But everything carries over to the real case with obvious modifications. All spaces shall be locally compact, Hausdorff, and second countable, and all $C^{*}$-algebras separable. We work equivariantly with respect to groupoids most of the time. We usually write $\mathcal{G}$ for a groupoid and $G$ for a group. All groupoids are - usually tacitly - required locally compact, Hausdorff, second countable, and with a Haar system (see [30, Definition 2.2]). We do not consider non-Hausdorff groupoids because they have few proper actions on Hausdorff spaces.

Let $\mathcal{G}$ be a groupoid (as above). We write $Z:=\mathcal{G}^{(0)}$ for its object space, $\mathcal{G}^{(1)}$ for its morphism space, and $r, s: \mathcal{G}^{(1)} \rightrightarrows \mathcal{G}^{(0)}$ for the range and source maps. By our convention, the spaces $\mathcal{G}^{(1)}$ and $\mathcal{G}^{(0)}$ are locally compact, Hausdorff, second countable, and the range, source, unit, multiplication and inversion maps are continuous. The existence of a Haar system forces the range, source, and multiplication maps to be open (see 37] or [25, Proposition 2.2.1]).

Actions of groupoids on spaces and $C^{*}$-algebras are explained, for instance, in 21, which is the basic reference for Kasparov theory for groupoids. We will use this theory and its main properties throughout, without much comment.

Recall that a space over $\mathcal{G}^{(0)}$ is a space with a continuous map to $\mathcal{G}^{(0)}$. A $\mathcal{G}$-space is a space over $\mathcal{G}^{(0)}$ with maps $\alpha_{g}: X_{s(g)} \rightarrow X_{r(g)}$ for all $g \in G$, where $X_{s(g)}$ and $X_{r(g)}$ are the fibres of $X$ over $s(g)$ and $r(g)$; the maps $\alpha_{g}$ vary continuously in the sense that they combine to a continuous map from $s^{*}(X):=\mathcal{G}^{(1)} \times{ }_{s} X$ to $r^{*}(X):=\mathcal{G}^{(1)} \times{ }_{s} X$.

Crossed products for groupoid actions on $C^{*}$-algebras are defined in great generality in 31]. We denote the $C^{*}$-algebra crossed product by $\mathcal{G} \ltimes A$ and groupoid $C^{*}$-algebras by $C^{*}(\mathcal{G})$. Readers unfamiliar with this may restrict attention to the well-known case of group actions and their crossed products. Since we only consider proper actions here, full and reduced crossed products agree in all cases we need.

Given an action of a groupoid $\mathcal{G}$ on a space $X$, we often form the crossed product groupoid $\mathcal{G} \ltimes X$, which is another groupoid with object space $X$ and morphisms encoding the action. We have a canonical $C^{*}$-algebra isomorphism

$$
C^{*}(\mathcal{G} \ltimes X) \cong \mathcal{G} \ltimes \mathcal{C}_{0}(X)
$$

because both algebras satisfy the same universal property.

Let $\mathcal{G}$ be a (locally compact, Hausdorff, etc.) groupoid and $X$ a proper $\mathcal{G}$-space. Although the following definitions make sense for non-proper actions as well, we only consider the special case of proper actions in this article.

Definition 2.2. The $\mathcal{G}$-equivariant $\mathrm{K}$-theory of $X$ is defined by

$$
\mathrm{K}_{\mathcal{G}}^{*}(X):=\mathrm{K}_{*}\left(\mathcal{G} \ltimes \mathcal{C}_{0}(X)\right) \cong \mathrm{K}_{*}\left(C^{*}(\mathcal{G} \ltimes X)\right) ;
$$

here we use the canonical isomorphism (2.1).

If $\mathcal{G}$ is a group, then $\mathrm{K}_{\mathcal{G}}^{*}(X)$ agrees with the equivariant $\mathrm{K}$-theory for locally compact groups studied by Chris Phillips in [28. We will soon spell out a Kasparov type description of $\mathrm{K}_{\mathcal{G}}^{*}(X)$ using Fredholm operators on continuous fields of Hilbert spaces, which is more similar to Phillips' definition. Moreover, Definition 2.2 is a special case of the definition of twisted equivariant K-theory in [35].

Definition 2.3. The $\mathcal{G}$-equivariant representable $\mathrm{K}$-theory of $X$ is

$$
\operatorname{RK}_{\mathcal{G}}^{*}(X):=\mathrm{KK}_{*}^{\mathcal{G} \ltimes X}\left(\mathcal{C}_{0}(X), \mathcal{C}_{0}(X)\right) .
$$

Definition 2.4. Let $Y$ be a $\mathcal{G} \ltimes X$-space via some map $p: Y \rightarrow X$. A subset $A$ of $Y$ is $X$-compact if $p: Y \rightarrow X$ restricts to a proper map $A \rightarrow X$. 
Definition 2.5. Let $Y$ be a $\mathcal{G} \ltimes X$-space via some map $p: Y \rightarrow X$. The $\mathcal{G}$-equivariant $\mathrm{K}$-theory of $Y$ with $X$-compact support is

$$
\operatorname{RK}_{\mathcal{G}, X}^{*}(Y):=\mathrm{KK}_{*}^{\mathcal{G} \ltimes X}\left(\mathcal{C}_{0}(X), \mathcal{C}_{0}(Y)\right) .
$$

This definition contains $\operatorname{RK}_{\mathcal{G}}^{*}(X)=\mathrm{RK}_{\mathcal{G}, X}^{*}(X)$ as a special case. In contrast, $\mathrm{K}_{\mathcal{G}}^{*}(X)$ need not be a special case of Definition 2.5. Our terminology is justified by Theorem 3.8, which describes $\mathrm{RK}_{\mathcal{G}}^{0}(X), \mathrm{RK}_{\mathcal{G}, X}^{0}(Y)$, and $\mathrm{K}_{\mathcal{G}}^{0}(X)$ using spaces of maps that only differ by support conditions.

Definition 2.6. A $\mathcal{G}$-equivariant vector bundle over $X$ is a vector bundle $V$ over $X$ together with a continuous, fibrewise linear $\mathcal{G}$-action on its total space.

Isomorphism classes of $\mathcal{G}$-equivariant vector bundles over $X$ form a monoid $\operatorname{Vect}_{\mathcal{G}}(X)$ with respect to the usual direct sum of vector bundles. Let $\operatorname{VK}_{\mathcal{G}}^{0}(X)$ be the Grothendieck group of this monoid.

We can also extend Definition 2.6 to pairs of spaces. By definition, a pair of $\mathcal{G}$-spaces is a $\mathcal{G}$-space $X$ together with a closed $\mathcal{G}$-invariant subspace $A$.

Definition 2.7. Let $(X, A)$ be a pair of $\mathcal{G}$-spaces. Consider triples $\left(V^{+}, V^{-}, \varphi\right)$ where $V^{ \pm} \in \operatorname{Vect}_{\mathcal{G}}(X)$ and $\varphi:\left.\left.V^{+}\right|_{A} \rightarrow V^{-}\right|_{A}$ is a $\mathcal{G}$-equivariant vector bundle isomorphism. Isomorphism classes of such triples form a monoid with respect to direct sum, which we denote by $\operatorname{Vect}_{\mathcal{G}}(X, A)$.

Call a triple $\left(V^{+}, V^{-}, \varphi\right)$ degenerate if $\varphi$ extends to a $\mathcal{G}$-equivariant vector bundle isomorphism $V^{+} \rightarrow V^{-}$. The degenerate triples form a submonoid of $\operatorname{Vect}_{\mathcal{G}}(X, A)$. Call two triples stably isomorphic if they become isomorphic after adding degenerate triples. We let $\operatorname{VK}_{\mathcal{G}}^{0}(X, A)$ be the monoid of stable isomorphism classes of $\operatorname{Vect}_{\mathcal{G}}(X, A)$.

By design, the class of degenerate triples is a neutral element in $\operatorname{VK}_{\mathcal{G}}^{0}(X, A)$. For any triple $\left(V^{+}, V^{-}, \varphi\right)$, the direct $\operatorname{sum}\left(V^{+}, V^{-}, \varphi\right) \oplus\left(V^{-}, V^{+}, \varphi^{-1}\right)$ is degenerate (compare [9, Lemma 1.42]). Hence $\operatorname{VK}_{\mathcal{G}}^{0}(X, A)$ is a group. There is a canonical isomorphism $\operatorname{VK}_{\mathcal{G}}^{0}(X, \emptyset) \cong \mathrm{VK}_{\mathcal{G}}^{0}(X)$.

We extend $\mathrm{VK}_{\mathcal{G}}^{0}(X)$ to a graded theory by

$$
\mathrm{VK}_{\mathcal{G}}^{-n}(X):=\mathrm{VK}_{\mathcal{G}}^{0}\left(X \times \mathbb{S}^{n}, X \times\{\star\}\right),
$$

where $\mathbb{S}^{n}$ denotes the $n$-sphere and $\star$ is its base point. For the relative theory, we put

$$
\mathrm{VK}_{\mathcal{G}}^{-n}(X, A):=\operatorname{VK}_{\mathcal{G}}^{0}\left(X \times \mathbb{S}^{n}, X \times\{\star\} \cup A \times \mathbb{S}^{n}\right) .
$$

Remark 2.9. Let $G$ be a compact group. The Green-Julg Theorem ([16]) identifies $\mathrm{K}_{G}^{*}(X)$ with the equivariant K-theory of $X$ studied by topologists, and [17, Proposition 2.20] identifies $\operatorname{RK}_{G}^{*}(X)$ with the $G$-equivariant representable K-theory in the sense of Graeme Segal ([33]). This justifies our notation.

If both $G$ and $X$ are compact, then $\mathrm{K}_{G}^{*}(X) \cong \mathrm{RK}_{G}^{*}(X) \cong \operatorname{VK}_{G}^{*}(X)$. If $X$ is not compact, then $\mathrm{K}_{G}^{*}(X) \cong \mathrm{VK}_{G}^{*}\left(X^{+},\{\infty\}\right)$, where $X^{+}=X \cup\{\infty\}$ is the one-point compactification of $X$. There is a canonical map $\operatorname{VK}_{G}^{*}(X) \rightarrow \mathrm{RK}_{G}^{*}(X)$ for any $X$, which may or may not be an isomorphism.

\section{Description by MAPS to Fredholm OPERATORS}

Our next goal is to describe $\operatorname{RK}_{\mathcal{G}}^{0}(X)$ as the space of homotopy classes of equivariant maps to a suitable space of Fredholm operators. Such a description is well-known in the non-equivariant case, see [3, 33. For equivariant K-theory, this is a special case of a similar result for twisted equivariant K-theory in [35]. For general groupoids, we equip the space of Fredholm operators with an unusual topology because the norm topology does not make sense. In $\$ 3.5$ we restrict attention to 
groups, where the norm topology makes sense, and show that the result remains valid if we use the norm topology instead.

3.1. Regular representation and Equivariant Stabilisation Theorem. Let $\mathcal{G}$ be a (locally compact, Hausdorff, etc.) groupoid and let $Z:=\mathcal{G}^{(0)}$. For $z \in Z$, let

$$
\mathcal{G}_{z}:=\left\{g \in \mathcal{G}^{(1)} \mid s(g)=z\right\} .
$$

The Haar system of $\mathcal{G}$ consists of measures $\left(\lambda_{z}\right)_{z \in Z}$ such that $\lambda_{z}$ has support $\mathcal{G}_{z}$ and

$$
Z \ni z \mapsto \int_{\mathcal{G}_{z}} f(g) \mathrm{d} \lambda_{z}(g)
$$

is a continuous function for all $f \in \mathcal{C}_{c}\left(\mathcal{G}^{(1)}\right)$ and $\mathrm{d} \lambda_{g z}=g \cdot \mathrm{d} \lambda_{z}$ (see [30, Definition $2.2])$.

Define a $\mathcal{C}_{0}(Z)$-valued inner product on $\mathcal{C}_{c}\left(\mathcal{G}^{(1)}\right)$ by

$$
\left\langle f_{1}, f_{2}\right\rangle(z):=\int_{\mathcal{G}_{z}} \overline{f_{1}(g)} \cdot f_{2}(g) \mathrm{d} \lambda_{z}(g)
$$

and a right $\mathcal{C}_{0}(Z)$-module structure by pointwise multiplication. This yields a preHilbert module, whose completion is a Hilbert module over $\mathcal{C}_{0}(Z)$, which we denote by $L^{2}(\mathcal{G})$. The usual left regular representation of $\mathcal{G}$ on $\mathcal{C}_{c}\left(\mathcal{G}^{(1)}\right)$ is compatible with the pre-Hilbert module structure, so that $L^{2}(\mathcal{G})$ becomes a $\mathcal{G}$-equivariant Hilbert $\mathcal{C}_{0}(Z)$-module. This is the left regular representation of $\mathcal{G}$.

Definition 3.1. Let $A$ be a $\mathcal{G}$ - $C^{*}$-algebra. Then we define

$$
\mathcal{H}_{A, \mathcal{G}}:=L^{2}(\mathcal{G})^{\infty} \otimes_{\mathcal{C}_{0}(Z)} A,
$$

equipped with the diagonal representation of $\mathcal{G}$. Here we write $\mathcal{E}^{\infty}$ for the Hilbert module direct sum of countably many copies of a Hilbert module $\mathcal{E}$.

Theorem 3.2 (Equivariant Stabilisation Theorem, see [34, Corollaire 6.22]). Let $X$ be a proper $\mathcal{G}$-space, let $A$ be a $\mathcal{G} \ltimes X-C^{*}$-algebra, and let $\mathcal{E}$ be a countably generated $\mathcal{G}$-equivariant Hilbert $A$-module. Then there is a $\mathcal{G}$-equivariant isomorphism $\mathcal{E} \oplus$ $\mathcal{H}_{A, \mathcal{G}} \cong \mathcal{H}_{A, \mathcal{G}}$.

This usually allows us to restrict attention to the special Hilbert module $\mathcal{H}_{A, \mathcal{G}}$.

Hilbert modules over $\mathcal{C}_{0}(Z)$ correspond to continuous fields of Hilbert spaces over $Z$ in the sense of [10]. The continuous field corresponding to $L^{2}(\mathcal{G})$ has as its fibres the Hilbert spaces $L^{2}\left(\mathcal{G}_{z}, \mathrm{~d} \lambda_{z}\right)$ for $z \in Z$. The topology on $\bigsqcup_{z \in Z} L^{2}\left(\mathcal{G}_{z}, \mathrm{~d} \lambda_{z}\right)$ is defined so that $\mathcal{C}_{c}\left(\mathcal{G}^{(1)}\right)$ is dense in the resulting Banach space of $\mathcal{C}_{0}$-sections.

If $\mathcal{G}=G \ltimes X$ for a locally compact group $G$ and a locally compact $G$-space $X$, then

$$
L^{2}(G \ltimes X) \cong L^{2}(G) \otimes \mathcal{C}_{0}(X)
$$

with $G$ acting diagonally. Disregarding the group action, the continuous field of Hilbert spaces corresponding to $L^{2}(G \ltimes X)$ is the trivial bundle with fibre $L^{2}(G)$ everywhere. In contrast, for a general groupoid the continuous field of Hilbert spaces $L^{2}(\mathcal{G})$ need not even be locally trivial.

3.2. The space of Fredholm operators. We can now define the set of Fredholm operators we are interested in. We restrict attention to essentially unitary operators, that is, operators whose image in the Calkin algebra is unitary.

Definition 3.3. If $\mathcal{H}$ is a Hilbert space, then $\mathcal{F}_{\mathcal{H}}$ or $\mathcal{F}(\mathcal{H})$ denotes the set of contractive, essentially unitary operators on $\mathcal{H}$ :

$$
\mathcal{F}_{\mathcal{H}}:=\left\{T \in \mathbb{B}(\mathcal{H}) \mid\|T\| \leq 1, \quad T T^{*}-\operatorname{id}_{\mathcal{H}}, T^{*} T-\operatorname{id}_{\mathcal{H}} \in \mathbb{K}(\mathcal{H})\right\} .
$$


If $\mathcal{H}=\left(\mathcal{H}_{x}\right)_{x \in X}$ is a bundle of Hilbert spaces over some space $X$, we let

$$
\mathcal{F}_{\mathcal{H}}:=\bigsqcup_{x \in X} \mathcal{F}_{\mathcal{H}_{x}}
$$

equipped with the obvious map to $X$. Here $\bigsqcup$ denotes the disjoint union.

It is a subtle problem to topologise $\mathcal{F}_{\mathcal{H}}$. It may be impossible to define a good norm topology. To understand this, suppose first that our bundle of Hilbert spaces is trivial with fibre $\mathcal{H}_{0}$. Equivalently, we are dealing with the Hilbert module $\mathcal{C}_{0}\left(X, \mathcal{H}_{0}\right)$. Then $\mathcal{F}(\mathcal{H})=X \times \mathcal{F}\left(\mathcal{H}_{0}\right)$, and we may indeed equip $\mathcal{F}\left(\mathcal{H}_{0}\right)$ with the norm topology and then take the product topology on $\mathcal{F}(\mathcal{H})$. But this topology depends on the trivialisation.

Two trivialisations differ by a unitary operator on $\mathcal{C}_{0}\left(X, \mathcal{H}_{0}\right)$. Such unitary operators correspond to ${ }^{*}$-strongly continuous maps $U: X \rightarrow \mathcal{U}\left(\mathcal{H}_{0}\right)$, where $\mathcal{U}\left(\mathcal{H}_{0}\right)$ denotes the group of unitary operators on $\mathcal{H}_{0}$. The ${ }^{*}$-strong topology means that both $U(x) \xi$ and $U(x)^{*} \xi$ are continuous maps $X \rightarrow \mathcal{H}_{0}$ for any $\xi \in \mathcal{H}_{0}$. Unless $\mathcal{H}_{0}$ is finite-dimensional, there are ${ }^{*}$-strongly continuous maps $X \rightarrow \mathcal{U}\left(\mathcal{H}_{0}\right)$ that are not norm-continuous. Twisting our trivialisation by such a map yields a different norm topology on $\mathcal{F}_{\mathcal{H}}$.

As a result, the norm topology on $\mathcal{F}_{\mathcal{H}}$ is only well-defined if we have a preferred trivialisation to start with. While this is clearly the case for groupoids of the form $G \ltimes X$ for a group $G$, the bundle of Hilbert spaces $L^{2}(\mathcal{G})$ need not even be locally trivial in general.

In order to construct a more canonical topology on $\mathcal{F}_{\mathcal{H}}$, we first topologise bounded subsets in the trivial bundles $X \times \mathbb{B}\left(\mathcal{H}_{0}\right)$ and $X \times \mathbb{K}\left(\mathcal{H}_{0}\right)$ of bounded and compact operators.

On $X \times \mathbb{B}\left(\mathcal{H}_{0}\right)$, we take the ${ }^{*}$-strong topology. Thus a net $\left(x_{\alpha}, T_{\alpha}\right)$ in $X \times$ $\mathbb{B}\left(\mathcal{H}_{0}\right)$ converges towards $(x, T)$ if and only if $\lim x_{\alpha}=x$ and $\lim T_{\alpha} \xi=T \xi$ and $\lim T_{\alpha}^{*} \xi=\xi$ for all $\xi \in \mathcal{H}_{0}$. As long as $\left(T_{\alpha}\right)$ is bounded, this topology is invariant under left or right pointwise multiplication with ${ }^{*}$-strongly continuous maps $X \rightarrow$ $\mathcal{U}\left(\mathcal{H}_{0}\right)$. We have restricted attention to Fredholm operators with $\|T\| \leq 1$ because the multiplication is jointly continuous for the ${ }^{*}$-strong topology only on bounded subsets of $\mathbb{B}\left(\mathcal{H}_{0}\right)$.

On $X \times \mathbb{K}\left(\mathcal{H}_{0}\right)$, we take the norm topology, that is, a net $\left(x_{\alpha}, T_{\alpha}\right)$ in $X \times \mathbb{K}\left(\mathcal{H}_{0}\right)$ converges towards $(x, T)$ if and only if $\lim x_{\alpha}=x$ and $\lim \left\|T_{\alpha}-T\right\|=0$. The crucial point is that on compact operators the norm topology is invariant under multiplication by ${ }^{*}$-strongly continuous maps $U: X \rightarrow \mathcal{U}\left(\mathcal{H}_{0}\right)$. For this, we must show that a net $\left(x_{\alpha}, U\left(x_{\alpha}\right) \cdot T_{\alpha}\right)$ in $X \times \mathbb{K}\left(\mathcal{H}_{0}\right)$ converges to $(x, U(x) \cdot T)$ if $\left(x_{\alpha}, T_{\alpha}\right)$ converges to $(x, T)$. We estimate

$$
\begin{aligned}
\left\|U\left(x_{\alpha}\right) T_{\alpha}-U(x) T\right\| & \leq\left\|U\left(x_{\alpha}\right)\left(T_{\alpha}-T\right)\right\|+\left\|\left(U\left(x_{\alpha}\right)-U(x)\right) T\right\| \\
& \leq\left\|T_{\alpha}-T\right\|+\left\|\left(U\left(x_{\alpha}\right)-U(x)\right) T\right\| .
\end{aligned}
$$

The first term goes to 0 by assumption. To see that the second one goes to 0 , we take $\varepsilon>0$ and split $T=F+K$ with a finite-rank operator $F$ and $\|K\|<\varepsilon$. Then

$$
\begin{aligned}
\left\|\left(U\left(x_{\alpha}\right)-U(x)\right) T\right\| & \leq\left\|\left(U\left(x_{\alpha}\right)-U(x)\right) F\right\|+\left\|\left(U\left(x_{\alpha}\right)-U(x)\right) K\right\| \\
& \leq\left\|\left(U\left(x_{\alpha}\right)-U(x)\right) F\right\|+2 \varepsilon
\end{aligned}
$$

The first term goes to 0 because $F$ has finite rank and $U\left(x_{\alpha}\right) \rightarrow U(x)$ strongly.

As a result, the norm topology on $X \times \mathbb{K}\left(\mathcal{H}_{0}\right)$ is invariant under left multiplication by *-strongly continuous families of unitary operators. A similar argument deals with right multiplication. 
So far, we have only considered operators on trivial Hilbert modules. Now let $\mathcal{E}$ be any countably generated Hilbert $\mathcal{C}_{0}(X)$-module. By the (non-equivariant) Kasparov Stabilisation Theorem, there exists an adjointable isometry

$$
V: \mathcal{E} \stackrel{\text { can }}{\longrightarrow} \mathcal{E} \oplus \mathcal{C}_{0}\left(X, \ell^{2} \mathbb{N}\right) \cong \mathcal{C}_{0}\left(X, \ell^{2} \mathbb{N}\right)
$$

View $\mathcal{E}$ as a continuous field of Hilbert spaces over $X$ with fibres $\mathcal{E}_{x}$ for $x \in X$. Then $V$ restricts to isometries on the fibres $V(x): \mathcal{E}_{x} \rightarrow \ell^{2} \mathbb{N}$.

Definition 3.4. The ${ }^{*}$-strong topology on $\bigsqcup \mathbb{B}\left(\mathcal{E}_{x}\right)$ is defined as follows: a net $\left(x_{\alpha}, T_{\alpha}\right)$ converges in $\bigsqcup \mathbb{B}\left(\mathcal{E}_{x}\right)$ if and only if $\left(x_{\alpha}, V\left(x_{\alpha}\right) T_{\alpha} V\left(x_{\alpha}\right)^{*}\right)$ converges in $X \times$ $\mathbb{B}\left(\ell^{2} \mathbb{N}\right)$ in the ${ }^{*}$-strong topology.

Similarly, the norm topology on $\bigsqcup \mathbb{K}\left(\mathcal{E}_{x}\right)$ is defined as follows: a net $\left(x_{\alpha}, T_{\alpha}\right)$ converges in $\bigsqcup \mathbb{K}\left(\mathcal{E}_{x}\right)$ if and only if $\left(x_{\alpha}, V\left(x_{\alpha}\right) T_{\alpha} V\left(x_{\alpha}\right)^{*}\right)$ converges in $X \times \mathbb{K}\left(\ell^{2} \mathbb{N}\right)$ in the norm topology.

If $W$ is another isometry as above, then there exists a unitary operator $U$ on $\mathcal{C}_{0}\left(X, \ell^{2} \mathbb{N}\right)$ with $W=U V$. Since the ${ }^{*}$-strong topology on the bounded operators and the norm topology on the compact operators are invariant under this change of trivialisation, the two topologies in Definition 3.4 are well-defined.

Lemma 3.5. The $C^{*}$-algebra of compact operators on $\mathcal{E}$ agrees with the $C^{*}$-algebra of norm-continuous sections of the bundle $\bigsqcup \mathbb{K}\left(\mathcal{E}_{x}\right)$ that vanish at infinity.

Proof. This is clear for trivial bundles of Hilbert spaces and follows in general from the Stabilisation Theorem.

Definition 3.6. Let $\mathcal{H}=\left(\mathcal{H}_{x}\right)_{x \in X}$ be a continuous field of Hilbert spaces over $X$. We equip the set $\mathcal{F}_{\mathcal{H}}=\bigsqcup_{x \in X} \mathcal{F}_{\mathcal{H}_{x}}$ with the topology defined by the map

$$
\mathcal{F}_{\mathcal{H}} \rightarrow \bigsqcup_{x \in X} \mathbb{B}\left(\mathcal{H}_{x}\right) \times \bigsqcup_{x \in X} \mathbb{K}\left(\mathcal{H}_{x}\right) \times \bigsqcup_{x \in X} \mathbb{K}\left(\mathcal{H}_{x}\right), \quad F \mapsto\left(F, 1-F F^{*}, 1-F^{*} F\right)
$$

That is, a net $\left(x_{\alpha}, F_{\alpha}\right)$ in $\mathcal{F}_{\mathcal{H}_{x}}$ converges to $(x, F)$ if and only if

- $x_{\alpha}$ converges to $x$,

- $F_{\alpha}$ converges to $F^{*}$-strongly,

- $1-F_{\alpha} F_{\alpha}^{*}$ converges to $1-F F^{*}$ in norm,

- $1-F_{\alpha}^{*} F_{\alpha}$ converges to $1-F^{*} F$ in norm.

A similar definition applies to the space of Fredholm operators between the fibres of two different continuous fields of Hilbert spaces.

Now we consider the continuous field of Hilbert spaces $L^{2}(\mathcal{G})^{\infty}$ over the object space $Z$ of a locally compact groupoid $\mathcal{G}$ with Haar system. We abbreviate

$$
\mathcal{F}_{\mathcal{G}}:=\mathcal{F}\left(L^{2}(\mathcal{G})^{\infty}\right) \text {. }
$$

Since $\mathcal{G}$ acts continuously on $L^{2}(\mathcal{G})^{\infty}$, the induced action of $\mathcal{G}$ on $\mathbb{K}\left(L^{2}(\mathcal{G})^{\infty}\right)$ by conjugation is continuous for the norm topology, and the induced action on the unit ball of $\mathbb{B}\left(L^{2}(\mathcal{G})^{\infty}\right)$ is strongly continuous and hence ${ }^{*}$-strongly continuous. (Here we also use that the multiplication map is jointly continuous for the strong topology on bounded subsets of $\mathbb{B}\left(L^{2}(\mathcal{G})^{\infty}\right)$.) Therefore, the induced action of $\mathcal{G}$ on $\mathcal{F}_{\mathcal{G}}$ by conjugation on the fibres is continuous in the topology described above.

Definition 3.7. Let $f: X \rightarrow \mathcal{F}_{\mathcal{H}}$ be a $\mathcal{G}$-equivariant continuous map. Its support is the closure of the set of $x \in X$ where $f(x)$ is not unitary.

Theorem 3.8. Let $\mathcal{G}$ be a locally compact groupoid with Haar system, form $\mathcal{F}_{\mathcal{G}}$ as above. There are natural isomorphisms between

- $\operatorname{RK}_{\mathcal{G}}^{0}(X)$ and the group of homotopy classes of $\mathcal{G}$-equivariant continuous maps $f: X \rightarrow \mathcal{F}_{\mathcal{G}}$; 
- $\mathrm{K}_{\mathcal{G}}^{0}(X)$ and the group of homotopy classes of $\mathcal{G}$-equivariant continuous maps $f: X \rightarrow \mathcal{F}_{\mathcal{G}}$ with $\mathcal{G}$-compact support;

- $\operatorname{RK}_{\mathcal{G}, Y}^{0}(X)$ and the group of homotopy classes of $\mathcal{G}$-equivariant continuous maps $f: X \rightarrow \mathcal{F}_{\mathcal{G}}$ with $Y$-compact support; here $Y$ is another $\mathcal{G}$-space and $p: X \rightarrow Y$ is a continuous $\mathcal{G}$-equivariant map.

The description of $\mathrm{K}_{\mathcal{G}}^{0}(X)$ in Theorem 3.8 is a special case of [35, Theorem 3.14], which describes equivariant twisted K-theory in terms of Fredholm operators.

3.3. Proof of the theorem. Let $p: X \rightarrow Y$ be a $\mathcal{G}$-space over $Y$. We are going to prove the assertion about $\mathrm{RK}_{\mathcal{G}, Y}^{0}(X)$ in Theorem 3.8. We first recall Kasparov's definition of

$$
\operatorname{RK}_{\mathcal{G}, Y}^{0}(X):=\operatorname{KK}_{0}^{\mathcal{G} \ltimes Y}\left(\mathcal{C}_{0}(Y), \mathcal{C}_{0}(X)\right) .
$$

A cycle for $\operatorname{RK}_{\mathcal{G}, Y}^{0}(X)$ consists of a $\mathbb{Z} / 2$-graded $\mathcal{G}$-equivariant Hilbert module $\mathcal{E}$ over $\mathcal{C}_{0}(X)$, a $\mathcal{G} \ltimes Y$-equivariant ${ }^{*}$-homomorphism $\varphi$ from $\mathcal{C}_{0}(Y)$ to the $C^{*}$-algebra of adjointable, grading preserving operators on $\mathcal{E}$, and a self-adjoint, odd, almost $\mathcal{G}$-equivariant operator $F$ on $\mathcal{E}$, such that $\left(F^{2}-1\right) \varphi(h)$ and $[F, \varphi(h)]$ are compact for all $h \in \mathcal{C}_{0}(Y)$. Two cycles give the same element in $\operatorname{RK}_{\mathcal{G}, Y}^{0}(X)$ if and only if they are homotopy equivalent, where homotopies are, by definition, cycles for $\operatorname{RK}_{\mathcal{G}, Y}^{0}(X \times[0,1])$.

The $\mathcal{G} \ltimes Y$-linearity completely determines $\varphi$ : it must be given by pointwise multiplication: $\varphi(f)(\xi)=\xi \cdot(f \circ p)$ for all $\xi \in \mathcal{E}, f \in \mathcal{C}_{0}(Y)$. Hence we can omit this part of the data and are left with a pair $(\mathcal{E}, F)$. Since $[F, \varphi(h)]=0$ for all $h \in \mathcal{C}_{0}(Y)$, we can also forget about one of the compactness conditions. Since $\mathcal{G}$ acts properly on $X$, any almost $\mathcal{G}$-equivariant operator on $\mathcal{E}$ has a compact perturbation that is exactly $\mathcal{G}$-equivariant (see [34, $\S 6.3]$ ). Therefore, we may restrict attention to cycles with $\mathcal{G}$-equivariant $F$. In addition, we can achieve $\|F\| \leq 1$ by functional calculus. We assume that this is the case from now on.

Let $\mathcal{E}^{ \pm}$be the even and odd subspaces of $\mathcal{E}$ with respect to the $\mathbb{Z} / 2$-grading. The block matrix decomposition of $F$ is

$$
F=\left(\begin{array}{cc}
0 & U^{*} \\
U & 0
\end{array}\right)
$$

for an adjointable operator $U: \mathcal{E}^{+} \rightarrow \mathcal{E}^{-}$with $\|U\| \leq 1$ because $F$ is odd, selfadjoint and contractive. Hence we may replace $(\mathcal{E}, F)$ by $\left(\mathcal{E}^{+}, \mathcal{E}^{-}, U\right)$, where $\mathcal{E}^{ \pm}$ are $\mathcal{G}$-equivariant Hilbert modules over $\mathcal{C}_{0}(X)$ and $U: \mathcal{E}^{+} \rightarrow \mathcal{E}^{-}$is a $\mathcal{G}$-equivariant adjointable operator of norm 1 , such that $\left(1-U^{*} U\right) \cdot \varphi(f \circ p)$ and $\left(1-U U^{*}\right) \cdot \varphi(f \circ p)$ are compact operators on $\mathcal{E}^{+}$and $\mathcal{E}^{-}$, respectively, for all $f \in \mathcal{C}_{0}(Y)$.

The Equivariant Stabilisation Theorem 3.2 asserts that there are $\mathcal{G}$-equivariant unitary operators

$$
\mathcal{E}^{ \pm} \oplus L^{2}(\mathcal{G} \ltimes X)^{\infty} \cong L^{2}(\mathcal{G} \ltimes X)^{\infty} .
$$

Since addition of the degenerate cycle id: $L^{2}(\mathcal{G} \ltimes X)^{\infty} \rightarrow L^{2}(\mathcal{G} \ltimes X)^{\infty}$ leads to a homotopic cycle, we may restrict attention to cycles whose underlying Hilbert module is $L^{2}(\mathcal{G} \ltimes X)^{\infty}$. Thus only the operator $U$ remains as data.

Lemma 3.9. Two cycles $U_{0}, U_{1}: L^{2}(\mathcal{G} \ltimes X)^{\infty} \rightarrow L^{2}(\mathcal{G} \ltimes X)^{\infty}$ for $\operatorname{RK}_{\mathcal{G}, Y}^{0}(X)$ have the same class in $\operatorname{RK}_{\mathcal{G}, Y}^{0}(X)$ if and only if there is a cycle $U$ on $L^{2}(\mathcal{G} \ltimes X \times[0,1])^{\infty}$ that restricts to $U_{0}$ and $U_{1}$ at the endpoints.

Proof. Let $\mathcal{H}:=L^{2}(\mathcal{G} \ltimes X) \otimes L^{2}([0,1])$, where we use the Lebesgue measure on $[0,1]$. This $\mathcal{G}$-equivariant Hilbert module over $\mathcal{C}_{0}(X)$ is unitarily equivalent to $L^{2}(\mathcal{G} \ltimes X)^{\infty}$, so that we may replace $U_{0}$ and $U_{1}$ by operators on $\mathcal{H}$. By the definition of our equivalence relation, the cycles $U_{0}$ and $U_{1}$ are homotopic via a homotopy that is realised on some $\mathcal{G}$-equivariant Hilbert module $\mathcal{E}$ over $\mathcal{C}_{0}(X \times[0,1])$. Using the 
Equivariant Stabilisation Theorem, we get a homotopy between $U_{0} \oplus \mathrm{id}$ and $U_{1} \oplus \mathrm{id}$ realised on the Hilbert module $\mathcal{E} \oplus \mathcal{H} \otimes \mathcal{C}([0,1]) \cong \mathcal{H} \otimes \mathcal{C}([0,1])$.

We identify $\mathcal{H} \cong \mathcal{H} \oplus \mathcal{H}$ using the unitary operator

$$
V: L^{2}([0,1])=L^{2}([0,1 / 2]) \oplus L^{2}([1 / 2,1]) \stackrel{S_{1} \oplus S_{2}}{\cong} L^{2}([0,1]) \oplus L^{2}([0,1]),
$$

where

$$
\begin{array}{ll}
S_{1}(f)(t):=\sqrt{2} f(t / 2) & \text { for } f \in L^{2}([0,1 / 2]), \\
S_{2}(f)(t):=\sqrt{2} f((t+1) / 2) & \text { for } f \in L^{2}([1 / 2,1]) .
\end{array}
$$

Conjugating by $V$, the homotopy above yields one between $V^{*}\left(U_{0} \oplus \operatorname{id}_{\mathcal{H}}\right) V$ and $V^{*}\left(U_{1} \oplus \operatorname{id}_{\mathcal{H}}\right) V$ that is realised on $\mathcal{H} \otimes \mathcal{C}([0,1])$. It remains, therefore, to find a homotopy between $V^{*}\left(U_{t} \oplus \operatorname{id}_{\mathcal{H}}\right) V$ and $U_{t}$. For this, we use the ${ }^{*}$-strongly continuous family of unitary operators

$$
V_{s}: L^{2}([0,1])=L^{2}([0, s]) \oplus L^{2}([s, 1]) \cong L^{2}([0,1]) \oplus L^{2}([0,1])
$$

for $s \in[1 / 2,1]$ defined like $S_{1} \oplus S_{2}$. The operators $V_{s}^{*}\left(U_{t} \oplus \operatorname{id}_{\mathcal{H}}\right) V_{s}$ for $t \in[0,1]$ provide the desired homotopy between $U_{t} \oplus \operatorname{id}_{\mathcal{H}}$ and $U_{t}$.

The homotopies in the proof of Lemma 3.9 are only ${ }^{*}$-strongly continuous. Such homotopies are much easier to accomplish than norm-continuous homotopies.

Now we identify $L^{2}(\mathcal{G} \ltimes X)^{\infty}$ with the space of $\mathcal{C}_{0}$-sections of a continuous field of Hilbert spaces over $X$ and replace $U: L^{2}(\mathcal{G} \ltimes X)^{\infty} \rightarrow L^{2}(\mathcal{G} \ltimes X)^{\infty}$ by a family $\left(U_{x}\right)_{x \in X}$ of operators between the fibres of this continuous field.

Since $\left(1-U U^{*}\right) \varphi(f \circ p)$ and $\left(1-U^{*} U\right) \varphi(f \circ p)$ are compact for all $f \in \mathcal{C}_{0}(Y)$, the operators $1-U_{x} U_{x}^{*}$ and $1-U_{x}^{*} U_{x}$ are compact for all $x \in X$. We also have $\left\|U_{x}\right\| \leq 1$ for all $x \in X$. Thus $x \mapsto U_{x}$ is a map from $X$ to $\mathcal{F}_{\mathcal{G}}$. The equivariance of $U$ is equivalent to the $\mathcal{G}$-equivariance of this map.

Since $\left(U_{x}\right)_{x \in X}$ defines an adjointable operator on $L^{2}(\mathcal{G} \ltimes X)^{\infty}$, the map $x \mapsto U_{x}$ is ${ }^{*}$-strongly continuous. Since $\left(1-U U^{*}\right) \varphi(f \circ p)$ and $\left(1-U^{*} U\right) \varphi(f \circ p)$ are compact for all $f \in \mathcal{C}_{0}(Y)$ and continuity is a local issue, the maps $x \mapsto 1-U_{x} U_{x}^{*}$ and $x \mapsto 1-U_{x}^{*} U_{x}$ are norm-continuous. Thus the map $x \mapsto U_{x}$ is continuous for the topology in Defition 3.6. Finally, the compactness of $\left(1-U U^{*}\right) \varphi(f \circ p)$ and $\left(1-U^{*} U\right) \varphi(f \circ p)$ implies that $\left\|\left(1-U_{x} U_{x}^{*}\right)\right\| \cdot f(p(x))$ and $\left\|\left(1-U_{x}^{*} U_{x}\right)\right\| \cdot f(p(x))$ are $\mathcal{C}_{0}$-functions on $X$. Equivalently, the subsets

$$
\left\{x \in X \mid\left\|1-U_{x}^{*} U_{x}\right\| \geq \varepsilon \text { or }\left\|1-U_{x} U_{x}^{*}\right\| \geq \varepsilon\right\}
$$

are $Y$-compact for all $\varepsilon>0$. This is not quite the support condition that we want, but we can improve this condition using functional calculus.

Let $f: \mathbb{R} \rightarrow[0,1]$ be a continuous function with $f(t)=t^{-1 / 2}$ for $t \geq 1 / 2$ and let $U^{\prime}:=U \cdot f\left(U^{*} U\right)$, so that $U_{x}^{\prime}=U_{x} \cdot f\left(U_{x}^{*} U_{x}\right)$ for all $x \in X$. This yields a homotopic cycle for $\operatorname{RK}_{\mathcal{G}, Y}^{*}(X)$ via a linear homotopy between $U$ and $U^{\prime}$ because $U$ is essentially unitary and $f(1)=1$. The operator $U_{x}^{\prime}$ is unitary where both $\left\|1-U_{x}^{*} U_{x}\right\| \leq 1 / 2$ and $\left\|1-U_{x} U_{x}^{*}\right\| \leq 1 / 2$. Hence $\left(U_{x}^{\prime}\right)_{x \in X}$ has $Y$-compact support.

Thus a cycle for $\operatorname{RK}_{\mathcal{G}, Y}^{0}(X)$ yields a $\mathcal{G}$-equivariant continuous map $X \rightarrow \mathcal{F}_{\mathcal{G}}$ with $Y$-compact support. Since we can apply the same construction to homotopies, Lemma 3.9 yields that homotopic cycles for $\mathrm{RK}_{\mathcal{G}, Y}^{0}(X)$ yield homotopic maps $X \rightarrow \mathcal{F}_{\mathcal{G}}$. Conversely, a $\mathcal{G}$-equivariant continuous map $X \rightarrow \mathcal{F}_{\mathcal{G} \ltimes X}$ with $Y$-compact support yields a cycle for $\operatorname{RK}_{\mathcal{G}, Y}^{0}(X)$, and homotopic maps yield homotopic cycles. Thus $\operatorname{RK}_{\mathcal{G}, Y}^{0}(X)$ agrees with the set of homotopy classes of $\mathcal{G}$-equivariant continuous maps $X \rightarrow \mathcal{F}_{\mathcal{G}}$ with $Y$-compact support as asserted in Theorem 3.8

The description of $\mathrm{RK}_{\mathcal{G}, Y}^{0}(X)$ contains $\mathrm{RK}_{\mathcal{G}}^{0}(X)=\mathrm{RK}_{\mathcal{G}, X}^{0}(X)$ as a special case; notice that if $p$ is the identity map on $X$, then any closed subset of $X$ is $X$-compact. Thus we get the assertion about $\operatorname{RK}_{\mathcal{G}}^{0}(X)$ in Theorem 3.8 
Now we turn to the group $\mathrm{K}_{\mathcal{G}}^{0}(X)$, which we describe as $\mathrm{KK}_{0}\left(\mathbb{C}, \mathcal{G} \ltimes \mathcal{C}_{0}(X)\right)$. Cycles for $\mathrm{KK}_{0}\left(\mathbb{C}, \mathcal{G} \ltimes \mathcal{C}_{0}(X)\right)$ consist of two Hilbert modules $\tilde{\mathcal{E}}^{ \pm}$over $\mathcal{G} \ltimes \mathcal{C}_{0}(X)$ together with an adjointable operator $\tilde{U}: \mathcal{E}^{+} \rightarrow \mathcal{E}^{-}$such that $1-\tilde{U} \tilde{U}^{*}$ and $1-\tilde{U}^{*} \tilde{U}$ are compact. The $C^{*}$-category of Hilbert modules over $\mathcal{G} \ltimes \mathcal{C}_{0}(X)$ is equivalent to the $C^{*}$-category of $\mathcal{G}$-equivariant Hilbert modules over $\mathcal{C}_{0}(X)$; this is implicit in 34 , Proposition 6.24]. Hence we may replace $\tilde{\mathcal{E}}^{ \pm}$by $\mathcal{G}$-equivariant Hilbert modules $\mathcal{E}^{ \pm}$ over $\mathcal{C}_{0}(X)$ and $\tilde{U}$ by a $\mathcal{G}$-equivariant adjointable operator $U: \mathcal{E}^{+} \rightarrow \mathcal{E}^{-}$. Applying the Equivariant Stabilisation Theorem 3.2 we reduce to $\mathcal{E}^{ \pm}=L^{2}(\mathcal{G} \ltimes X)^{\infty}$.

A $\mathcal{G}$-equivariant adjointable operator $T$ on $L^{2}(\mathcal{G} \ltimes X)^{\infty}$ corresponds to a compact operator on $C^{*}(\mathcal{G} \ltimes X)^{\infty}$ if and only if $T \cdot \varphi(c)$ is compact, where $c$ is a cut-off function. This is a variant of [34, Proposition 6.24]. Now the argument is essentially the same as above.

3.4. Comparing the support conditions. The descriptions of

$$
\operatorname{RK}_{\mathcal{G}}^{0}(X), \quad \operatorname{RK}_{\mathcal{G}, Y}^{0}(X), \quad \text { and } \quad \mathrm{K}_{\mathcal{G}}^{0}(X)
$$

in Theorem 3.8 differ only in the support conditions. This justifies calling $\operatorname{RK}_{\mathcal{G}, Y}^{0}(X)$ the K-theory of $X$ with $Y$-compact and $\mathrm{K}_{\mathcal{G}}^{0}(X)$ the K-theory of $X$ with $\mathcal{G}$-compact support, whereas $\mathrm{RK}_{\mathcal{G}}^{0}(X)$ is the $\mathrm{K}$-theory of $X$ without support restriction.

Proposition 3.10. There are canonical maps

$$
\mathrm{K}_{\mathcal{G}}^{*}(X) \rightarrow \mathrm{RK}_{\mathcal{G}, Y}^{*}(X) \rightarrow \mathrm{RK}_{\mathcal{G}}^{*}(X) .
$$

The first is an isomorphism if $Y$ is $\mathcal{G}$-compact. The second map is an isomorphism once the map $X \rightarrow Y$ is proper. Both maps are isomorphisms if $X$ is $\mathcal{G}$-compact.

A continuous $\mathcal{G}$-map $h: Y \rightarrow Y^{\prime}$ induces a map $\operatorname{RK}_{\mathcal{G}, Y^{\prime}}^{*}(X) \rightarrow \operatorname{RK}_{\mathcal{G}, Y}^{*}(X)$, which is an isomorphism if $h$ is proper.

Proof. It suffices to treat the even K-groups, where we can use Theorem 3.8 Thus it suffices to compare the various support conditions.

We claim that any $\mathcal{G}$-compact subset of $X$ is $Y$-compact. Let $K \subseteq X$ and $L \subseteq Y$ be compact and $g \in \mathcal{G}^{(1)}$, then $g \cdot K \cap p^{-1}(L) \neq \emptyset$ if and only if $g \cdot p(K) \cap L \neq \emptyset$; since $Y$ is proper, the set of $g \in \mathcal{G}^{(1)}$ for which this happens is compact, so that $\mathcal{G} \cdot K \cap p^{-1}(L)$ is compact. This means that $p$ restricts to a proper map on $\mathcal{G} \cdot K$, so that all $\mathcal{G}$-invariant closed subsets of $\mathcal{G} \cdot K$ are $Y$-compact.

Since $\mathcal{G}$-compact subsets of $X$ are $Y$-compact, we get a map $\mathrm{K}_{\mathcal{G}}^{0}(X) \rightarrow \mathrm{RK}_{\mathcal{G}, Y}^{0}(X)$. If $Y$ is $\mathcal{G}$-compact, say, $Y=\mathcal{G} \cdot L$, then any $Y$-compact $\mathcal{G}$-invariant subset $A$ of $X$ is $\mathcal{G}$-compact because it is contained in $\mathcal{G} \cdot\left(p^{-1}(L) \cap A\right)$. Therefore, $\mathrm{K}_{\mathcal{G}}^{0}(X) \cong$ $\mathrm{RK}_{\mathcal{G}, Y}^{0}(X)$ if $Y$ is $\mathcal{G}$-compact. If $X$ is $\mathcal{G}$-compact, the same argument shows that all our support conditions are vacuously satisfied, so that all three groups coincide by Theorem 3.8. Theorem 3.8 yields a canonical map $\mathrm{RK}_{\mathcal{G}, Y}^{0}(X) \rightarrow \mathrm{RK}_{\mathcal{G}}^{0}(X)$, forgetting the support restriction.

Finally, consider a map $h: Y \rightarrow Y^{\prime}$. Then any $Y^{\prime}$-compact subset of $X$ is also $Y$-compact, and the converse holds if and only if $h$ is proper. Therefore, we get a map $\operatorname{RK}_{\mathcal{G}, Y^{\prime}}^{0}(X) \rightarrow \mathrm{RK}_{\mathcal{G}, Y}^{0}(X)$, and it is an isomorphism if $h$ is proper.

3.5. Changing the topology. Now we restrict attention to group actions on spaces. In this case, we do have a canonical trivialisation

$$
L^{2}(G \ltimes X)^{\infty} \cong \mathcal{C}_{0}\left(X, L^{2}(G)^{\infty}\right),
$$

with $G$ acting diagonally. Hence the norm topology on the bundle of Fredholm operators is meaningful. In this section, we let $\mathcal{F}_{G}$ be the space of essentially unitary operators on $\mathcal{H}_{G}:=L^{2}(G)^{\infty}$, equipped with the norm topology. 
Theorem 3.11. Let $G$ be a locally compact group. There are natural isomorphisms between

- $\operatorname{RK}_{G}^{0}(X)$ and the group of homotopy classes of $G$-equivariant (norm) continuous maps $f: X \rightarrow \mathcal{F}_{G}$;

- $\mathrm{K}_{G}^{0}(X)$ and the group of homotopy classes of $G$-equivariant continuous maps $f: X \rightarrow \mathcal{F}_{G}$ with $G$-compact support;

- $\mathrm{RK}_{G, Y}^{0}(X)$ and the group of homotopy classes of $G$-equivariant continuous maps $f: X \rightarrow \mathcal{F}_{G}$ with $Y$-compact support; here $Y$ is a proper $\mathcal{G}$-space and $p: X \rightarrow Y$ is a G-equivariant continuous map.

Corollary 3.12. The space of norm-continuous $G$-equivariant maps from $X$ to the unitary group $\mathcal{U}\left(\mathcal{H}_{G}\right)$ is contractible.

Proof. Since maps $X \rightarrow \mathcal{F}_{G}$ that are unitary everywhere represent zero in $\operatorname{RK}_{G}^{0}(X)$, Theorem 3.11 shows that this space of maps is connected. Since we may replace $X$ by $X \times \mathbb{S}^{n}$ for $n \in \mathbb{N}$, this space is weakly contractible as well. We may replace unitaries by invertibles without changing the homotopy type. This yields an open subset of the $C^{*}$-algebra of $G$-equivariant norm-continuous maps $X \rightarrow \mathbb{B}\left(\mathcal{H}_{G}\right)$. Open subsets of Banach spaces have the homotopy type of a CW-complex by 20 , Corollary 5.5 in Chapter 4]. Hence they are contractible once they are weakly contractible.

The only difference between Theorems 3.8 and 3.11 is the topology on the bundle of Fredholm operators.

The proof of Theorem 3.11 will occupy the remainder of this section. We concentrate on the case $\mathrm{RK}_{G, Y}^{0}(X)$ because it contains $\mathrm{RK}_{G}^{0}(X)$ as a special case and $\mathrm{K}_{G}^{0}(X)$ is analogous.

The first part of the argument is similar to the proof of [12, Proposition 13]. Let $D_{\mathrm{s}}$ be the $C^{*}$-algebras of $G$-equivariant maps $X \rightarrow \mathbb{B}\left(\mathcal{H}_{G}\right)$ that are ${ }^{*}$-strongly continuous, and let $D_{\mathrm{n}} \subseteq D_{\mathrm{s}}$ be the subalgebra of norm-continuous maps.

Lemma 3.13. $\mathrm{K}_{*}\left(D_{\mathrm{n}}\right)=0$ and $\mathrm{K}_{*}\left(D_{\mathrm{s}}\right)=0$.

Proof. Both assertions are proved using an Eilenberg swindle. We carry this out only for $\mathrm{K}_{0}\left(D_{\mathrm{n}}\right)$ because the other three cases $\mathrm{K}_{1}\left(D_{\mathrm{n}}\right), \mathrm{K}_{0}\left(D_{\mathrm{s}}\right)$, and $\mathrm{K}_{1}\left(D_{\mathrm{s}}\right)$ are analogous. Since $D_{\mathrm{n}}$ is unital and matrix stable, it suffices to study idempotents in $D_{\mathrm{n}}$. Let $p$ be such an idempotent and write $p^{\perp}:=1-p$. Choose an isomorphism $\mathcal{H}_{G} \cong \mathcal{H}_{G}^{\infty}$ and use this to transform the diagonal operator $\infty \cdot p:=p \oplus p \oplus p \oplus p \oplus \cdots$ into an operator on $\mathcal{H}_{G}$. Notice that this is again a norm-continuous function $X \rightarrow \mathbb{B}\left(\mathcal{H}_{G}\right)$ if $p$ is. The projections $p \oplus \infty \cdot p$ and $0 \oplus \infty \cdot p$ are equivalent via the unilateral shift operator on $\mathcal{H}_{G}^{\infty}$, which we view as a constant function $X \rightarrow \mathbb{B}\left(\mathcal{H}_{G}^{\infty}\right)$. Hence $[p]=0$ in $\mathrm{K}_{0}\left(D_{\mathrm{n}}\right)$.

Let $J_{0}$ be the algebra of norm-continuous $G$-equivariant maps $X \rightarrow \mathbb{K}\left(\mathcal{H}_{G}\right)$ whose support is $Y$-compact. This is a (non-closed) ${ }^{*}$-ideal in both $D_{\mathrm{n}}$ and $D_{\mathrm{s}}$. Its closure is the $C^{*}$-algebra $J$ of all norm-continuous $\mathcal{G}$-equivariant maps $f: X \rightarrow \mathbb{K}\left(\mathcal{H}_{G}\right)$ for which the subsets $\{x \in X \mid\|f(x)\|>\varepsilon\}$ are $Y$-compact for all $\varepsilon>0$.

Let $Q_{\mathrm{n}}$ and $Q_{\mathrm{s}}$ be the quotients of $D_{\mathrm{n}}$ and $D_{\mathrm{s}}$ by the ideal $J$, respectively.

Lemma 3.14. $\mathrm{K}_{*+1}\left(Q_{\mathrm{n}}\right) \cong \mathrm{K}_{*+1}\left(Q_{\mathrm{s}}\right) \cong \operatorname{RK}_{G, Y}^{*}(X)$.

Proof. The embedding $D_{\mathrm{n}} \rightarrow D_{\mathrm{s}}$ induces a morphism of $C^{*}$-algebra extensions

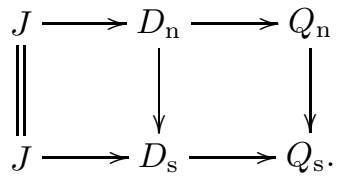


The vertical maps $J \rightarrow J$ and $D_{\mathrm{n}} \rightarrow D_{\mathrm{s}}$ induce isomorphisms on K-theory by Lemma 3.13. Now the Five Lemma applied to the long exact K-theory sequence yields $\mathrm{K}_{*}\left(Q_{\mathrm{n}}\right) \cong \mathrm{K}_{*}\left(Q_{\mathrm{s}}\right)$.

For the second isomorphism, we only prove $\mathrm{K}_{1}\left(Q_{\mathrm{s}}\right) \cong \operatorname{RK}_{G, Y}^{0}(X)$, the other parity can be reduced to this by suspension. The $C^{*}$-algebra $Q_{\mathrm{s}}$ is unital and matrix stable, that is, $Q_{\mathrm{s}} \cong \mathbb{M}_{n}\left(Q_{\mathrm{s}}\right)$. Hence any cycle for $\mathrm{K}_{1}\left(Q_{\mathrm{s}}\right)$ is represented by a unitary element in $Q_{\mathrm{s}}$. A lifting for such a unitary element to $D_{\mathrm{s}}$ is nothing but an adjointable operator $U$ on the Hilbert module $\mathcal{C}_{0}\left(X, \mathcal{H}_{G}\right)$ with

$$
1-U U^{*} \in J \quad \text { and } \quad 1-U^{*} U \in J .
$$

These are exactly the cycles that describe $\operatorname{RK}_{G, Y}^{0}(X)$.

The equivalence relation on $\mathrm{K}_{1}\left(Q_{\mathrm{s}}\right)$ is generated by stabilisation - replacing $U$ by $V\left(U \oplus \operatorname{id}_{\mathcal{H}_{G}}\right) V^{*}$ for a unitary $V: \mathcal{H}_{G}^{2} \rightarrow \mathcal{H}_{G}$ - and norm-continuous homotopy in $U$. In Kasparov theory, these two relations are called addition of degenerate cycles and operator homotopy, and are known to generate the same equivalence relation as homotopy (in the non-equivariant case, this is [4, Theorem 18.5.3]; the same argument works for equivariant Kasparov theory). Hence $\mathrm{K}_{1}\left(Q_{\mathrm{s}}\right) \cong \operatorname{RK}_{G, Y}^{0}(X)$.

The group $\mathrm{K}_{1}\left(Q_{\mathrm{n}}\right)$ can be described similarly. The only difference to $\mathrm{K}_{1}\left(Q_{\mathrm{s}}\right)$ is that its cycles are norm continuous $G$-equivariant maps $U: X \rightarrow \mathcal{F}_{G}$. As in the proof of Theorem 3.8 , we can restrict attention to maps $f$ with $Y$-compact support. The equivalence relation on $\mathrm{K}_{1}\left(Q_{\mathrm{s}}\right)$ is generated by two moves: normcontinuous homotopy and stabilisation. It remains to prove, therefore, that for any norm-continuous $G$-equivariant map $U: X \rightarrow \mathcal{F}_{G}$ with $Y$-compact support, there is a norm-continuous homotopy between $U$ and $V\left(U \oplus \operatorname{id}_{\mathcal{H}_{G}}\right) V^{*}$ for the standard unitary $V: \mathcal{H}_{G}^{2} \rightarrow \mathcal{H}_{G}$.

As in more classical situations ([15]), this follows from a suitable generalisation of Kuiper's Theorem (see Corollary 3.12 ). In our case, we need to know that the unitary group of $D_{\mathrm{n}}$ is contractible. The most general form of Kuiper's Theorem in the literature is due to Joachim Cuntz and Nigel Higson ([8, see also [36, §16] for a more detailed proof); it asserts that the unitary group of a stable multiplier algebra $\mathcal{M}(A \otimes \mathbb{K})$ is norm-contractible for any $\sigma$-unital $C^{*}$-algebra $A$. Unfortunately, this does not cover the algebra $D_{\mathrm{n}}$ we need: only $D_{\mathrm{s}}$ is a stable multiplier algebra. Nevertheless, inspection shows that the argument in 8 can be carried over to $D_{\mathrm{n}}$. We concentrate on those steps in the argument that require change. We modify the structure of the argument slightly to simplify the application to Fredholm operators, which is not considered in 8 .

We fix an isometry $V=\left(V_{1} V_{2}\right): \mathcal{H}_{G}^{2} \rightarrow \mathcal{H}_{G}$ as above, that is, $V_{1}$ and $V_{2}$ are isometries with $V_{1} V_{1}^{*}+V_{2} V_{2}^{*}=1$, and let $P:=V_{2} V_{2}^{*}$. We view $P, V_{1}, V_{2}$ as constant functions on $X$ and thus as elements of $D_{\mathrm{n}}$.

Lemma 3.15. Let $\bar{U} \in Q_{\mathrm{n}}$ be unitary. There are a lifting $U$ of $\bar{U}$ and isometries $S$ and $T$ in $D_{\mathrm{n}}$ such that:

- $U S$ is an isometry, and $U S$ and $U\left(1-S S^{*}\right)$ have orthogonal range;

- $V_{2}^{*} S=0$ and $V_{2}^{*} T=0$, that is, the range projections of $S$ and $T$ are orthogonal to $P$;

- $\left\|T^{*} U S\right\|<1$.

Before we prove this technical lemma, we use it to finish the proof of Theorem [3.11] As in [8], we call two projections $P$ and $Q$ in a unital $C^{*}$-algebra $A$ strongly equivalent and write $P \approx Q$ if there is a continuous path of unitaries $U \in \mathcal{C}([0,1], A)$ with $U_{0}=1_{A}$ and $U_{1} P U_{1}^{*}=Q$. Two projections are strongly equivalent if and only if they are homotopic ([4, Proposition 4.3.3]). [8, Lemma 1] 
asserts that two projections $P$ and $Q$ with $\|P Q\|<1$ are strongly equivalent once they are equivalent.

Let $R_{S}, R_{U S}$, and $R_{T}$ be the range projections of the isometries $S, U S$, and $T$, respectively. Recall that $P$ is the range projection of $V_{2}$. As range projections of isometries, these projections are all equivalent to 1 . Since $P R_{S}=P R_{T}=0$ and $\left\|R_{T} R_{U S}\right\|<1$ by Lemma 3.15, [8, Lemma 1] yields strong equivalences

$$
R_{S} \approx P, \quad R_{U S} \approx R_{T} \approx P .
$$

Thus we get continuous paths of unitaries $W_{1} \in \mathcal{C}\left([0,1], D_{\mathrm{n}}\right)$ and $W_{2} \in \mathcal{C}\left([0,1], D_{\mathrm{n}}\right)$ with $W_{1}(0)=W_{2}(0)=1$ and

$$
W_{1}(1) R_{U S} W_{1}(1)^{*}=P, \quad W_{2}(1) R_{S} W_{2}(1)^{*}=P .
$$

This provides a homotopy between $U$ and $U^{\prime}:=W_{1}(1) U W_{2}(1)^{*}$. By construction,

$$
\begin{aligned}
U^{\prime} P=W_{1}(1) U W_{2}(1)^{*} P= & W_{1}(1) U R_{S} W_{2}(1)^{*} \\
& =W_{1}(1) R_{U S} U W_{2}(1)^{*}=P W_{1}(1) U W_{2}(1)^{*}=P U^{\prime} .
\end{aligned}
$$

Hence

$$
U^{\prime}=V\left(\begin{array}{cc}
U_{1} & 0 \\
0 & U_{2}
\end{array}\right) V^{*}
$$

for the unitary $V: \mathcal{H}_{G}^{2} \rightarrow \mathcal{H}_{G}$ chosen above, with essentially unitary $U_{1}$ and $U_{2}$. The usual rotation homotopy that proves the commutativity of $K_{1}$ shows that $V\left(U_{1} \oplus U_{2}\right) V^{*}$ is homotopic to $V\left(U_{1} U_{2} \oplus 1\right) V^{*}$. But if two unitaries of the latter form are stably homotopic, then they are already homotopic because we can subsume any additional stabilisation in the summand 1 .

Hence $\mathrm{K}_{1}\left(Q_{\mathrm{n}}\right)$ is the set of homotopy classes of unitaries in $Q_{\mathrm{n}}$, without need to stabilise. Thus $\operatorname{RK}_{G}^{0}(X) \cong \mathrm{K}_{1}\left(Q_{\mathrm{n}}\right) \cong \mathrm{K}_{1}\left(Q_{\mathrm{s}}\right) \cong\left[X, \mathcal{F}_{G}\right]$, as desired.

To finish the proof of Theorem 3.11, it remains to prove Lemma 3.15.

Splitting $\ell^{2} \mathbb{N} \cong \ell^{2} \mathbb{N} \otimes \ell^{2} \mathbb{N}$, we can embed $\mathbb{B}\left(\ell^{2} \mathbb{N}\right) \otimes D_{\mathrm{n}}$ into $D_{\mathrm{n}}$. We choose an infinite orthonormal sequence of rank-one projections $\left(e_{n}\right)_{n \in \mathbb{N}}$ on $\ell^{2} \mathbb{N}$ such that $\sum e_{n} \otimes 1=V_{1} V_{1}^{*}$. Next we choose an approximate unit for the ideal $J \subseteq D_{\text {n }}$ with $u_{n+1} u_{n}=u_{n}$ for all $n \in \mathbb{N}$ that is locally uniformly continuous as a set of functions on $X$. We omit the proof that such an approximate unit exists. We view $e_{n} \otimes u_{n}$ as elements of $D_{\mathrm{n}}$. By construction, $\left(e_{n} \otimes u_{n}\right) P=0$ for all $n \in \mathbb{N}$. The local uniform continuity of $\left(u_{n}\right)$ is needed for the following analogue of [8, Lemma 3]:

Lemma 3.16. Let $\left(u_{n}\right)_{n \in \mathbb{N}}$ and $\left(e_{n}\right)_{n \in \mathbb{N}}$ be as above, and let $k: \mathbb{N} \rightarrow \mathbb{N}$ be an increasing function with $\lim k(n)=\infty$, so that $\left(u_{k(n)}\right)$ and $\left(e_{k(n)}\right)$ are subsequences of $\left(u_{n}\right)$ and $\left(e_{n}\right)$. Let $a:=\sum_{n \in \mathbb{N}} e_{k(n)} \otimes u_{k(n)}$. Then there is an isometry $T \in D_{\mathrm{n}}$ with $a T=T$ and hence $a T T^{*}=T T^{*}$ and $T T^{*} \leq a$.

Proof. The proof is identical to the proof of [8, Lemma 3]. The assumption on $u_{n}$ implies that the sequence $\left(d_{n}\right)$ defined by $d_{n}:=\left(u_{k(n-1)}-u_{k(n-2)}\right)^{1 / 2}$ is locally uniformly continuous, so that the sum $T:=\sum_{n \in \mathbb{N}}^{\infty} v_{n} \otimes d_{n}$ used in [8] is normcontinuous; here $\left(v_{n}\right)$ is a sequence of partial isometries with carefully selected range and source projections.

of Lemma 3.15. Choose any lifting $U_{0}$ of $\bar{U}$. First we modify $U_{0}$ and find an isometry $S_{0}$ to get the first property in the lemma. Let $\left(e_{n}\right)$ and $\left(u_{n}\right)$ be as above, and let $g_{n}:=e_{n} \otimes u_{n}$, viewed as elements of $D_{n}$. The sequence $\left(g_{n}\right)$ converges strictly to 0 because $\left(e_{n}\right) \rightarrow 0$ in the strict topology on $\mathbb{B}\left(\ell^{2} \mathbb{N}\right)$. Since $1-U_{0}^{*} U_{0} \in J$, we can find a subsequence $k(n)$ with

$$
\left\|\left(1-U_{0}^{*} U_{0}\right) g_{k(n)}\right\|<\varepsilon 2^{-n-1} .
$$


Lemma 3.16 yields an isometry $S_{0}$ with $\sum g_{k(n)} \cdot S_{0}=S_{0}$. Since $g_{n} P=\left(e_{n} \otimes u_{n}\right) P=$ 0 for all $n \in \mathbb{N}$, the range of $S_{0}$ is orthogonal to $P$ as desired. We have

$$
\left\|\left(1-U_{0}^{*} U_{0}\right) S_{0}\right\|=\left\|\sum\left(1-U_{0}^{*} U_{0}\right) g_{l(n)} S_{0}\right\| \leq \sum \varepsilon 2^{-n-1}=\varepsilon .
$$

By functional calculus, we can replace $U_{0}$ by another lifting $U$ of $\bar{U}$ such that $U S_{0}$ is exactly isometric and the range of $U\left(1-S_{0} S_{0}^{*}\right)$ is orthogonal to the range of $U S_{0}$.

Next we construct $T$ and an isometry $S_{1}$ such that $S:=S_{0} S_{1}$ and $T$ have all the required properties. Let $\left(g_{n}\right)$ be as above and let $h_{n}:=\left(U S_{0}\right) g_{n}\left(U S_{0}\right)^{*}$. We have already observed that $\left(g_{n}\right)$ converges strictly to zero in $\mathcal{M}(J)$. So does $h_{n}$ because multiplication in $\mathcal{M}(J)$ is separately strictly continuous. Therefore, $\left\|g_{m} h_{n}\right\|$ converges to 0 if either variable is fixed and the other goes to $\infty$. Thus we can find subsequences $k(n)$ and $l(n)$ such that $\left\|g_{k(m)} h_{l(n)}\right\| \leq \varepsilon 2^{-m-n-1}$. Lemma 3.16 provides isometries $S_{1}$ and $T$ with

$$
\sum g_{l(n)} \cdot S_{1}=S_{1}, \quad \sum g_{k(n)} \cdot T=T .
$$

As above, the range of $T$ is orthogonal to $P$. Moreover,

$$
T^{*} U S_{0} S_{1}=T^{*} \sum_{m} g_{k(m)} U S_{0} \sum_{n} g_{l(n)} S_{1}=T^{*} \sum_{m, n} g_{k(m)} h_{l(n)} U S_{0} S_{1}
$$

has norm at most $\sum \varepsilon 2^{-n-m-1}=\varepsilon$. Thus the isometries $S:=S_{0} S_{1}$ and $T$ have the desired properties.

\section{Representable K-theory via Crossed products}

As before, $\mathcal{G}$ is a locally compact, second countable, Hausdorff groupoid with Haar system and $X$ and $Y$ are proper, locally compact, second countable $\mathcal{G}$-spaces.

Our next goal is to describe $\mathrm{RK}_{\mathcal{G}}^{*}(X)$ and $\mathrm{RK}_{\mathcal{G}, Y}^{*}(X)$ as $\mathrm{K}$-theory groups of certain crossed products. The relevant crossed products are not $C^{*}$-algebras but $\sigma-C^{*}$-algebras (see [26]), and we use the K-theory for such algebras defined by Chris Phillips in 29]; he calls this theory "representable K-theory" to distinguish it from other theories like algebraic K-theory, which may be different even for $\mathrm{K}_{0}$. We drop the adjective because Phillips' theory is the only one with good homological properties for such topological algebras. The familiar properties of K-theory for $C^{*}$-algebras like Bott periodicity, stability, homotopy invariance, and six-term exact sequences for extensions all extend (see [29, Theorem 3.4]). We will use this in 44.1 to study the homological properties of the functor $\mathrm{RK}_{\mathcal{G}}^{*}$.

Definition 4.1 (see [26]). A $\sigma-C^{*}$-algebra is a complete topological ${ }^{*}$-algebra whose topology is defined by an increasing sequence of $C^{*}$-seminorms. Equivalently, it is a countable projective limit of $C^{*}$-algebras.

The next theorem is a generalisation of [12, Lemma 20] and [11, Theorem 2.8] to groupoids; the first result of this kind seems [18, Theorem 5.4]. For a better perspective, we have stated a more general theorem than we actually need, which involves bivariant $\mathrm{K}$-theory for $\sigma$ - $C^{*}$-algebras. Although it took some time to notice this, Kasparov theory extends literally to separable $\sigma$ - $C^{*}$-algebras. This was first carried out by Alexander Bonkat in his thesis [5, using the extension of Kasparov's Technical Lemma in [14. Bonkat's definition is equivalent to the obvious one suggested already by Chris Phillips in [29, p. 470]. The Kasparov group $\mathrm{KK}_{*}(\mathbb{C}, B)$ most relevant to us is defined in [29, Definition 4.1] and identified with Phillips' K-theory in [29, Theorem 4.2].

Theorem 4.2. Let $\left(X_{n}\right)$ be an increasing sequence of $\mathcal{G}$-compact, $\mathcal{G}$-invariant subsets of $X$ with $\bigcup X_{n}=X$. Let $A$ be a $C^{*}$-algebra with the trivial action of $\mathcal{G}$ and 
let $B$ be a $\mathcal{G} \ltimes X-C^{*}$-algebra. Then there is a natural isomorphism

$$
\mathrm{KK}_{*}^{\mathcal{G} \ltimes X}\left(\mathcal{C}_{0}(X) \otimes A, B\right) \cong \mathrm{KK}_{*}\left(A,\left.\underset{n}{\lim _{n}} \mathcal{G} \ltimes B\right|_{X_{n}}\right)
$$

If $X$ is $\mathcal{G}$-compact, then this agrees with $\operatorname{KK}_{*}(A, \mathcal{G} \ltimes B)$.

Any $\mathcal{G}$-compact, $\mathcal{G}$-invariant subset of $X$ is contained in $X_{n}$ for some $n \in \mathbb{N}$, and the subsets $X_{n}$ are automatically closed because the action is proper. Therefore, the restriction $\left.B\right|_{X_{n}}$ is simply the quotient of $B$ by the ideal $\mathcal{C}_{0}\left(X \backslash X_{n}\right) \cdot B$. The quotient maps turn $\left(\left.\mathcal{G} \ltimes B\right|_{X_{n}}\right)_{n \in \mathbb{N}}$ into a countable projective system of $C^{*}$-algebras with surjective maps, whose projective limit is a $\sigma$ - $C^{*}$-algebra.

All choices of the sequence $\left(X_{n}\right)$ are equivalent in a suitable sense and therefore yield isomorphic $\sigma$-C $C^{*}$-algebras $\left.\lim _{n} \mathcal{G} \ltimes B\right|_{X_{n}}$. In particular, if $X$ itself is $\mathcal{G}$-compact, then the right hand side agrees with the usual $\operatorname{Kasparov}_{\text {group }} \mathrm{KK}_{*}(A, \mathcal{G} \ltimes B)$.

We mainly use the case $B=\mathcal{C}_{0}(X)$, where we get $\left.B\right|_{X_{n}} \cong \mathcal{C}_{0}\left(X_{n}\right)$. The $\sigma-C^{*}$ algebra $\lim \mathcal{C}_{0}\left(X_{n}\right)$ consists of continuous functions $X \rightarrow \mathbb{C}$ whose restrictions to $X_{n}$ vanish at infinity for all $n \in \mathbb{N}$.

Notation 4.3. We abbreviate

$$
\sigma-C^{*}(\mathcal{G} \ltimes X):=\lim _{\longleftarrow} C^{*}\left(\mathcal{G} \ltimes X_{n}\right) \cong \lim \mathcal{G} \ltimes \mathcal{C}_{0}\left(X_{n}\right) .
$$

of Theorem 4.2. Let $\mathcal{G} \backslash X$ be the orbit space. [34, Proposition 6.25] yields

$$
\mathrm{KK}_{*}^{\mathcal{G} \ltimes X}\left(\mathcal{C}_{0}(X) \otimes A, B\right) \cong \mathrm{KK}_{*}^{\mathcal{G} \backslash X}\left(\mathcal{C}_{0}(\mathcal{G} \backslash X) \otimes A, \mathcal{G} \ltimes B\right) .
$$

Combining this with the non-equivariant case of [12, Lemma 20] yields the assertion. Alternatively, the proof of [12, Lemma 20] extends to the groupoid case.

Corollary 4.4. There is a natural isomorphism

$$
\operatorname{RK}_{\mathcal{G}}^{*}(X) \cong \mathrm{K}_{*}\left(\sigma-C^{*}(\mathcal{G} \ltimes X)\right) .
$$

If $X$ is $\mathcal{G}$-compact, then $\operatorname{RK}_{\mathcal{G}}^{*}(X) \cong \mathrm{K}_{*}\left(C^{*}(\mathcal{G} \ltimes X)\right)=\mathrm{K}_{\mathcal{G}}^{*}(X)$.

Corollary 4.5. Let $p: X \rightarrow Y$ be a continuous $\mathcal{G}$-equivariant map between two proper $\mathcal{G}$-spaces, and let $\left(Y_{n}\right)_{n \in \mathbb{N}}$ be an increasing sequence of $\mathcal{G}$-compact, $\mathcal{G}$-invariant subspaces of $Y$. Let $X_{n}:=p^{-1}\left(Y_{n}\right)$. Then there is a natural isomorphism

$$
\operatorname{RK}_{\mathcal{G}, Y}^{*}(X) \cong \mathrm{K}_{*}\left(\lim _{\longleftarrow} \mathcal{G} \ltimes \mathcal{C}_{0}\left(X_{n}\right)\right) \cong \mathrm{K}_{*}\left(\lim _{\longleftarrow} C^{*}\left(\mathcal{G} \ltimes X_{n}\right)\right) .
$$

The $\sigma$ - $C^{*}$-algebra $\lim C^{*}\left(\mathcal{G} \ltimes X_{n}\right)$ in Corollary 4.5 may differ from $\sigma$ - $C^{*}(\mathcal{G} \ltimes X)$ because the subsets $\overleftarrow{X_{n}}$ in Corollary 4.5 need not be $\mathcal{G}$-compact.

Both corollaries follow directly from the definitions using Theorem 4.2, The $C^{*}$-algebras $\mathcal{G} \ltimes \mathcal{C}_{0}\left(X_{n}\right)$ and $C^{*}\left(\mathcal{G} \ltimes X_{n}\right)$ are isomorphic by (2.1). We can also derive the results in $\$ 3.4$ from these corollaries. For instance, we get once again that $\operatorname{RK}_{\mathcal{G}, Y}^{*}(X)=\mathrm{K}_{\mathcal{G}}^{*}(X)$ if $Y$ is $\mathcal{G}$-compact.

Phillips' representable K-theory for $\sigma$ - $C^{*}$-algebras is computable by a Milnor $\lim ^{1}$-sequence $([29$, Theorem 3.2]). This specialises to short exact sequences

$$
\lim ^{1} \mathrm{~K}_{\mathcal{G}}^{*+1}\left(X_{n}\right) \longmapsto \mathrm{RK}_{\mathcal{G}, Y}^{*}(X) \rightarrow \lim _{\mathscr{G}} \mathrm{K}_{\mathcal{G}}^{*}\left(X_{n}\right),
$$

where $\left(X_{n}\right)$ is as in Corollary 4.5. Thus we can, in principle, reduce our theories to $\mathrm{K}_{\mathcal{G}}^{*}\left(X_{n}\right)$. 
4.1. Functorial properties. First we discuss multiplication. The Kasparov product turns

$$
\operatorname{RK}_{\mathcal{G}}^{*}(X):=\mathrm{KK}^{\mathcal{G} \ltimes X}\left(\mathcal{C}_{0}(X), \mathcal{C}_{0}(X)\right)
$$

into a graded-commutative ring with unit. This ring acts by exterior product on all Kasparov groups of the form $\mathrm{KK}^{\mathcal{G} \ltimes Z}(A, B)$ for $\mathcal{G} \ltimes X-C^{*}$-algebras $A$ and $B$, where $Z$ is another proper $\mathcal{G}$-space with a map $p: X \rightarrow Z$; we view $\mathcal{G} \ltimes X$ - $C^{*}$-algebras as $\mathcal{G} \ltimes Z$ - $C^{*}$-algebras via the resulting forgetful map. In particular, the ring $\operatorname{RK}_{\mathcal{G}}^{*}(X)$ acts on $\mathrm{RK}_{\mathcal{G}, Y}^{*}(X)$, that is, the latter is a graded module over $\mathrm{RK}_{\mathcal{G}}^{*}(X)$ in a canonical way. Furthermore, via the descent homomorphism

$$
\begin{aligned}
\operatorname{RK}_{\mathcal{G}}^{*}(X) & :=\mathrm{KK}^{\mathcal{G} \ltimes X}\left(\mathcal{C}_{0}(X), \mathcal{C}_{0}(X)\right) \\
& \stackrel{\text { descent }}{\longrightarrow} \mathrm{KK}\left(\mathcal{G} \ltimes \mathcal{C}_{0}(X), \mathcal{G} \ltimes \mathcal{C}_{0}(X)\right)=\mathrm{KK}\left(C^{*}(\mathcal{G} \ltimes X), C^{*}(\mathcal{G} \ltimes X)\right),
\end{aligned}
$$

the ring $\mathrm{RK}_{\mathcal{G}}^{*}(X)$ acts on $\mathrm{K}_{\mathcal{G}}^{*}(X):=\mathrm{K}_{*}\left(C^{*}(\mathcal{G} \ltimes X)\right)$. Thus $\mathrm{K}_{\mathcal{G}}^{*}(X)$ is a graded module over the graded ring $\mathrm{RK}_{\mathcal{G}}^{*}(X)$. Via the canonical map $\mathrm{K}_{\mathcal{G}}^{*}(X) \rightarrow \mathrm{RK}_{\mathcal{G}}^{*}(X)$, we may also equip $\mathrm{K}_{\mathcal{G}}^{*}(X)$ with a ring structure in its own right, but this ring need not be unital.

Now we turn to functoriality. We only discuss $\operatorname{RK}_{\mathcal{G}}^{*}(X)$ and $\mathrm{K}_{\mathcal{G}}^{*}(X)$.

A continuous $\mathcal{G}$-equivariant map $f: X \rightarrow Y$ induces a grading preserving ring homomorphism

$$
\operatorname{RK}_{\mathcal{G}}^{*}(f): \operatorname{RK}_{\mathcal{G}}^{*}(Y) \rightarrow \operatorname{RK}_{\mathcal{G}}^{*}(X)
$$

regardless whether $f$ is proper. Hence $X \mapsto \mathrm{RK}_{\mathcal{G}}^{*}(X)$ is a contravariant functor from the category of locally compact proper $\mathcal{G}$-spaces to the category of gradedcommutative graded rings. Similarly, a continuous, proper, $\mathcal{G}$-equivariant map induces a grading preserving group homomorphism

$$
\mathrm{K}_{\mathcal{G}}^{*}(f): \mathrm{K}_{\mathcal{G}}^{*}(Y) \rightarrow \mathrm{K}_{\mathcal{G}}^{*}(X) .
$$

Both functors $\mathrm{RK}_{\mathcal{G}}^{*}$ and $\mathrm{K}_{\mathcal{G}}^{*}$ are homotopy invariant (the latter only for proper homotopies, of course) because $\mathrm{K}$-theory for $\sigma-C^{*}$-algebras is homotopy invariant.

More generally, any continuous groupoid homomorphism (functor) $f: \mathcal{G} \ltimes X \rightarrow$ $\mathcal{G}^{\prime} \ltimes X^{\prime}$ induces maps

$$
f^{*}: \mathrm{KK}^{\mathcal{G}^{\prime} \ltimes X^{\prime}}(A, B) \rightarrow \mathrm{KK}^{\mathcal{G} \ltimes X}\left(f^{*} A, f^{*} B\right)
$$

by [21, $\S 7.1]$, where $f^{*} A:=\mathcal{C}_{0}(X) \otimes_{\mathcal{C}_{0}\left(X^{\prime}\right)} A$ equipped with the canonical action of $\mathcal{G}$. These are compatible with Kasparov products and satisfy the functoriality properties $(f \circ g)^{*}=g^{*} \circ f^{*}$ and $\mathrm{id}^{*}=\mathrm{id}$ as expected. Since $f^{*} \mathcal{C}_{0}\left(X^{\prime}\right) \cong \mathcal{C}_{0}(X)$, this specialises to a ring homomorphism $\mathrm{RK}_{\mathcal{G}^{\prime}}^{*}\left(X^{\prime}\right) \rightarrow \mathrm{RK}_{\mathcal{G}}^{*}(X)$, that is, equivariant representable $\mathrm{K}$-theory is functorial for strict groupoid morphisms.

Theorem 4.7. A Morita equivalence between the two groupoids $\mathcal{G} \ltimes X$ and $\mathcal{G}^{\prime} \ltimes X^{\prime}$ induces isomorphisms $\operatorname{RK}_{\mathcal{G}}^{*}(X) \cong \mathrm{RK}_{\mathcal{G}^{\prime}}^{*}\left(X^{\prime}\right)$ and $\mathrm{K}_{\mathcal{G}}^{*}(X) \cong \mathrm{K}_{\mathcal{G}^{\prime}}^{*}\left(X^{\prime}\right)$.

Proof. Morita equivalent groupoids give rise to equivalent Kasparov categories by [21, §7.2]. In particular, this yields the isomorphism $\operatorname{RK}_{\mathcal{G}}^{*}(X) \cong \operatorname{RK}_{\mathcal{G}^{\prime}}^{*}\left(X^{\prime}\right)$. Furthermore, Morita equivalent groupoids have Morita equivalent $C^{*}$-algebras by [24]. Therefore, $\mathrm{K}_{\mathcal{G}}^{*}(X) \cong \mathrm{K}_{\mathcal{G}^{\prime}}^{*}\left(X^{\prime}\right)$ as well.

Example 4.8. Let $G$ be a group and let $H$ be a closed subgroup in $G$. Then $G \ltimes G / H$ is Morita equivalent to $H$. Therefore, we have canonical induction isomorphisms

$$
\operatorname{RK}_{G}^{*}\left(G \times_{H} X\right) \cong \mathrm{RK}_{H}^{*}(X), \quad \mathrm{K}_{G}^{*}\left(G \times_{H} X\right) \cong \mathrm{K}_{H}^{*}(X)
$$

for any $H$-space $X$. 
Example 4.9. Let $X$ be a free and proper $\mathcal{G}$-space. Then $\mathcal{G} \ltimes X$ is Morita equivalent to the orbit space $\mathcal{G} \backslash X$. Hence

$$
\operatorname{RK}_{\mathcal{G}}^{*}(X) \cong \operatorname{RK}^{*}(\mathcal{G} \backslash X), \quad \mathrm{K}_{\mathcal{G}}^{*}(X) \cong \mathrm{K}^{*}(\mathcal{G} \backslash X) .
$$

Proposition 4.10. Let $\left(X_{n}\right)_{n \in \mathbb{N}}$ be $\mathcal{G}$-spaces and let $X:=\bigsqcup_{n \in \mathbb{N}} X_{n}$ be their disjoint union. Then

$$
\mathrm{K}_{\mathcal{G}}^{*}(X) \cong \bigoplus_{n \in \mathbb{N}} \mathrm{K}_{\mathcal{G}}^{*}\left(X_{n}\right), \quad \operatorname{RK}_{\mathcal{G}}^{*}(X) \cong \prod_{n \in \mathbb{N}} \mathrm{RK}_{\mathcal{G}}^{*}\left(X_{n}\right)
$$

Proof. The first isomorphism follows from the additivity of K-theory for direct sums of $C^{*}$-algebras. The second isomorphism follows from the behaviour of K-theory for $\sigma-C^{*}$-algebras for direct products (see [29, Proposition 3.1]).

Finally, we turn to exact sequences. To formulate them, we extend our theories to pairs of spaces $(X, A)$, where $X$ is a proper $\mathcal{G}$-space and $A \subseteq X$ is a closed $\mathcal{G}$-invariant subspace.

For $\mathrm{K}_{\mathcal{G}}^{*}$, we simply put

$$
\mathrm{K}_{\mathcal{G}}^{*}(X, A):=\mathrm{K}_{\mathcal{G}}^{*}(X \backslash A)=\mathrm{K}_{*}\left(\mathcal{G} \ltimes \mathcal{C}_{0}(X \backslash A)\right) .
$$

This extends the old theory because $\mathrm{K}_{\mathcal{G}}^{*}(X)=\mathrm{K}_{\mathcal{G}}^{*}(X, \emptyset)$. The excision property

$$
\mathrm{K}_{\mathcal{G}}^{*}(X, A) \cong \mathrm{K}_{\mathcal{G}}^{*}(X \backslash U, A \backslash U)
$$

for an open, $\mathcal{G}$-invariant subset $U \subseteq A$ is trivial. Since the (full) crossed product functor is exact, we get an extension of $C^{*}$-algebras

$$
\mathcal{G} \ltimes \mathcal{C}_{0}(X \backslash A) \longmapsto \mathcal{G} \ltimes \mathcal{C}_{0}(X) \rightarrow \mathcal{G} \ltimes \mathcal{C}_{0}(A)
$$

for any pair $(X, A)$, leading to an exact sequence

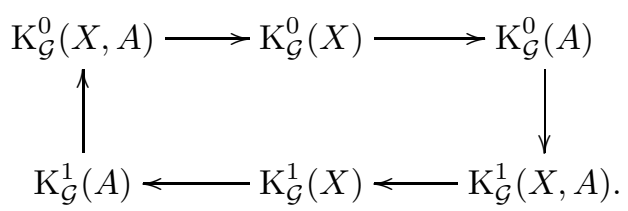

Pairs of $\mathcal{G}$-spaces form a category, whose morphisms are $\mathcal{G}$-equivariant continuous proper maps that restrict to maps between the specified subspaces. It is clear that $\mathrm{K}_{\mathcal{G}}^{*}$ is a contravariant functor on this category. The isomorphism in (4.12) is induced by the obvious morphism $(X \backslash U, A \backslash U) \rightarrow(X, A)$, the horizontal maps in (4.13) are induced by the obvious morphisms $(A, \emptyset) \rightarrow(X, \emptyset) \rightarrow(X, A)$.

For $\mathrm{RK}_{\mathcal{G}}^{*}$, we put

$$
\operatorname{RK}_{\mathcal{G}}^{*}(X, A):=\operatorname{RK}_{\mathcal{G}, X}^{*}(X \backslash A) .
$$

Corollary 4.5 identifies this with $\mathrm{K}_{*}\left(\lim _{\longleftarrow} \mathcal{G} \mathcal{C}_{0}\left(X_{n} \backslash A\right)\right)$, where $\left(X_{n}\right)$ is an increasing sequence of $\mathcal{G}$-compact $\mathcal{G}$-invariant subsets of $X$ with $\bigcup X_{n}=X$. Again, we have $\operatorname{RK}_{\mathcal{G}}^{*}(X)=\mathrm{RK}_{\mathcal{G}}^{*}(X, \emptyset)$. The excision property

$$
\operatorname{RK}_{\mathcal{G}}^{*}(X, A) \cong \operatorname{RK}_{\mathcal{G}}^{*}(X \backslash U, A \backslash U)
$$

holds for any open, $\mathcal{G}$-invariant subset $U \subseteq A$ because both sides are computed by the same $\sigma-C^{*}$-algebra. As above, we get a sequence of $C^{*}$-algebra extensions

$$
\mathcal{G} \ltimes \mathcal{C}_{0}\left(X_{n} \backslash A\right) \longmapsto \mathcal{G} \ltimes \mathcal{C}_{0}\left(X_{n}\right) \rightarrow \mathcal{G} \ltimes \mathcal{C}_{0}\left(X_{n} \cap A\right)
$$


for all $n \in \mathbb{N}$. The vertical maps

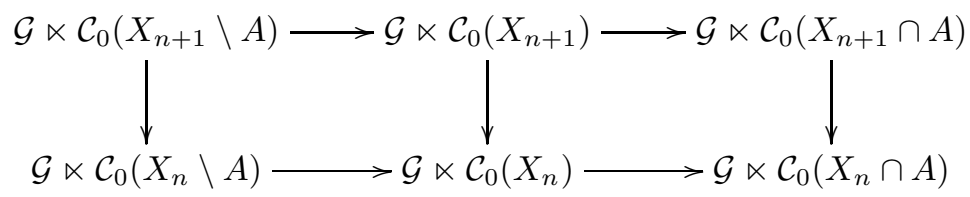

are all surjective. Therefore, we get an extension of $\sigma-C^{*}$-algebras

$$
\lim _{\longleftarrow} \mathcal{G} \ltimes \mathcal{C}_{0}\left(X_{n} \backslash A\right) \longmapsto \sigma-C^{*}\left(\mathcal{G} \ltimes X_{n}\right) \rightarrow \sigma-C^{*}\left(\mathcal{G} \ltimes X_{n} \cap A\right),
$$

which induces a six-term exact sequence

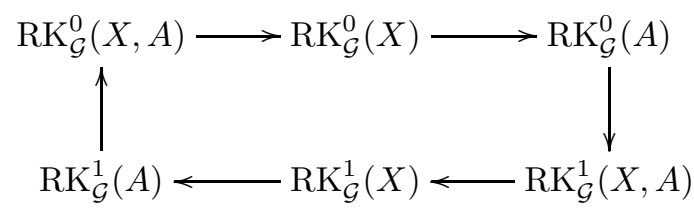

by [29, Theorem 3.4]. A $\mathcal{G}$-equivariant continuous map of pairs $(X, A) \rightarrow\left(X^{\prime}, A^{\prime}\right)$ induces a map $\mathrm{RK}_{\mathcal{G}}^{*}\left(X^{\prime}, A^{\prime}\right) \rightarrow \mathrm{RK}_{\mathcal{G}}^{*}(X, A)$ even if it is not proper. The horizontal maps in (4.16) and the excision isomorphism in (4.15) can be described as for $\mathrm{K}_{\mathcal{G}}^{*}$.

We define a relative version of $\mathrm{RK}_{\mathcal{G}, Y}^{*}$ by

$$
\operatorname{RK}_{\mathcal{G}, Y}^{*}(X, A):=\operatorname{RK}_{\mathcal{G}, Y}^{*}(X \backslash A)
$$

for a pair $(X, A)$ of $\mathcal{G} \ltimes Y$-spaces. Like $\mathrm{K}_{\mathcal{G}}^{*}$, this theory is functorial for proper morphisms of pairs, satisfies excision, and has long exact sequences.

The natural transformations $\mathrm{K}_{\mathcal{G}}^{*}(X) \rightarrow \mathrm{RK}_{\mathcal{G}, Y}^{*}(X) \rightarrow \mathrm{RK}_{\mathcal{G}}^{*}(X)$ extend to natural transformations

$$
\mathrm{K}_{\mathcal{G}}^{*}(X, A) \rightarrow \mathrm{RK}_{\mathcal{G}, Y}^{*}(X, A) \rightarrow \mathrm{RK}_{\mathcal{G}}^{*}(X, A) .
$$

The first of these maps is invertible if $Y$ is $\mathcal{G}$-compact, the second is invertible if the closure of $X \backslash A$ in $X$ is $Y$-compact: in both cases, the assumption ensures that both sides are computed by the same $\sigma$ - $C^{*}$-algebra.

Lemma 4.17. Any $\mathcal{G}$-space $X$ over $Y$ is of the form $X=\bar{X} \backslash \partial X$ for some $Y$-compact $\mathcal{G}$-space $\bar{X}$ and some closed $\mathcal{G}$-invariant subset $\partial X$ of $\bar{X}$, necessarily $Y$-compact. We have

$$
\operatorname{RK}_{\mathcal{G}, Y}^{*}(X) \cong \operatorname{RK}_{\mathcal{G}}^{*}(\bar{X}, \partial X) .
$$

Proof. The second assertion is just (4.14), so that it remains to construct $\bar{X}$ and $\partial X$. Roughly speaking, we construct $\bar{X}$ by one-point compactifying the fibres $p^{-1}(y) \subseteq X$ of the map $p: X \rightarrow Y$ for all $y \in Y$. More precisely, we let $\bar{X}:=X \sqcup Y$ with the unique topology where $X$ is open and carries the given topology, and $U_{X} \sqcup U_{Y} \subseteq$ $X \sqcup Y$ is a neighbourhood of $y \in Y$ if and only if $U_{Y}$ is a neighbourhood of $y$ in $Y$ and there is a neighbourhood $V$ of $y$ in $Y$ such that $p^{-1}(V) \backslash U_{X}$ is relatively compact. It is left to the reader to check that $\bar{X}$ is a locally compact Hausdorff space, that the map $\bar{X} \rightarrow Y$ is continuous and proper, and that the $\mathcal{G}$-action on $\bar{X}$ is continuous and proper.

For the special choice of $\bar{X}$ and $\partial X$ in the proof of Lemma 4.17, $\partial X=Y$ is a retract of $\bar{X}$ via the projection map $\bar{X} \rightarrow Y$. Hence the extension of $\sigma$ - $C^{*}$-algebras

$$
\lim _{\longleftarrow} \mathcal{G} \ltimes \mathcal{C}_{0}\left(X_{n}\right) \longmapsto \sigma-C^{*}\left(\mathcal{G} \ltimes \bar{X}_{n}\right) \rightarrow \sigma-C^{*}\left(\mathcal{G} \ltimes \partial X_{n}\right)
$$

splits. The resulting $\mathrm{K}$-theory long exact sequence splits as well, that is,

$$
\operatorname{RK}_{\mathcal{G}, Y}^{*}(X) \cong \operatorname{RK}_{\mathcal{G}}^{*}(\bar{X}, \partial X) \cong \operatorname{ker}\left(\operatorname{RK}_{\mathcal{G}}^{*}(\bar{X}) \rightarrow \operatorname{RK}_{\mathcal{G}}^{*}(\partial X)\right)
$$

This is analogous to the familiar description of $\mathrm{K}_{0}$ for non-unital $C^{*}$-algebras as $\mathrm{K}_{0}(A):=\operatorname{ker}\left(\mathrm{K}_{0}\left(A^{+}\right) \rightarrow \mathrm{K}_{0}(\mathbb{C})\right)$. 
Theorem 4.19. Let $X$ be a space over $Y$ and let $I_{Y}$ be the directed set of all relatively $Y$-compact, $\mathcal{G}$-invariant, open subsets of $X$ (that is, sets in $I_{Y}$ have $Y$-compact closure in $X)$. Then

$$
\mathrm{RK}_{\mathcal{G}, Y}^{*}(X) \cong \underset{A \in \lim _{Y}}{\operatorname{RK}_{\mathcal{G}, Y}^{*}}(A) \cong \lim _{A \in I_{Y}} \operatorname{RK}_{\mathcal{G}}^{*}(X, X \backslash A) .
$$

Similarly, let $I_{\mathcal{G}}$ be the directed set of all relatively $\mathcal{G}$-compact, $\mathcal{G}$-invariant, open subsets of $X$. Then

$$
\mathrm{K}_{\mathcal{G}}^{*}(X) \cong \lim _{A \in I_{\mathcal{G}}} \mathrm{K}_{\mathcal{G}}^{*}(A) \cong \lim _{\overrightarrow{A \in I_{\mathcal{G}}}} \mathrm{RK}_{\mathcal{G}}^{*}(X, X \backslash A)
$$

Proof. The isomorphism $\mathrm{K}_{\mathcal{G}}^{*}(X) \cong \lim _{A \in I_{\mathcal{G}}} \mathrm{K}_{\mathcal{G}}^{*}(A)$ follows because

$$
\mathcal{G} \ltimes \mathcal{C}_{0}(X) \cong \lim _{A \in I_{\mathcal{G}}} \mathcal{G} \ltimes \mathcal{C}_{0}(A)
$$

and K-theory for $C^{*}$-algebras is continuous with respect to direct limits. If $A \in I_{\mathcal{G}}$, then (4.11), the $\mathcal{G}$-compactness of $\bar{A}$, and (4.15) yield

$$
\mathrm{K}_{\mathcal{G}}^{*}(A) \cong \mathrm{K}_{\mathcal{G}}^{*}(\bar{A}, \bar{A} \backslash A) \cong \operatorname{RK}_{\mathcal{G}}^{*}(\bar{A}, \bar{A} \backslash A) \cong \operatorname{RK}_{\mathcal{G}}^{*}(X, X \backslash A) .
$$

We have an isomorphism

$$
\lim _{n \in \mathbb{N}} \mathcal{G} \ltimes \mathcal{C}_{0}\left(X_{n}\right) \cong \lim _{A \in I_{Y}} \lim _{n \in \mathbb{N}} \mathcal{G} \ltimes \mathcal{C}_{0}\left(A \cap X_{n}\right)
$$

as well, where the subsets $X_{n} \subseteq X$ are defined as in Corollary 4.5. But inductive limits of $\sigma-C^{*}$-algebras require some care because they may become uncountable projective systems of $C^{*}$-algebras. Hence we prefer another argument in this case.

Theorem 3.8 identifies $\mathrm{RK}_{\mathcal{G}, Y}^{*}(X)$ with the set of homotopy classes of continuous $\mathcal{G}$-equivariant maps $U: X \rightarrow \mathcal{F}_{\mathcal{G}}$ with $Y$-compact support. Any $Y$-compact subset of $X$ has a relatively $Y$-compact open neighbourhood. Let $A$ be such a neighbourhood of the support of $U$. The restriction of $U$ to $A$ is a cycle for $\operatorname{RK}_{\mathcal{G}, Y}^{*}(A)$. Similarly, if two cycles for $\operatorname{RK}_{\mathcal{G}, Y}^{*}(X)$ are homotopic, then we can find a relatively $Y$-compact neighbourhood $B$ for the support of the resulting homotopy and get a homotopy in $\operatorname{RK}_{\mathcal{G}, Y}^{*}(B)$. It remains to check that the canonical map $\mathrm{RK}_{\mathcal{G}, Y}^{*}(A) \rightarrow \mathrm{RK}_{\mathcal{G}, Y}^{*}(X)$ maps these restricted cycles to the original cycles. We must describe, therefore, how the latter map acts on maps $A \rightarrow \mathcal{F}_{\mathcal{G}}$.

Let $U: A \rightarrow \mathcal{F}_{\mathcal{G}}$ be a $\mathcal{G}$-equivariant continuous map whose support is closed in $X$. View $U$ as a $\mathcal{G}$-equivariant adjointable operator on the Hilbert module $L^{2}(\mathcal{G} \ltimes A)^{\infty}$ over $\mathcal{C}_{0}(A)$. Since $\mathcal{C}_{0}(A) \subseteq \mathcal{C}_{0}(X)$ is an ideal, we may view $L^{2}(\mathcal{G} \ltimes A)^{\infty}$ as a Hilbert module over $\mathcal{C}_{0}(X)$ and $U$ as an adjointable operator of Hilbert $\mathcal{C}_{0}(X)$-modules. If $U$ is the restriction of a map $\bar{U}: X \rightarrow \mathcal{F}_{\mathcal{G}}$ with the same support, then the cycle defined by $\bar{U}$ on $L^{2}(\mathcal{G} \ltimes X)^{\infty}$ is homotopic to the one defined by $U$ on $L^{2}(\mathcal{G} \ltimes A)^{\infty}$ : the homotopy is given by the $\mathcal{G}$-equivariant Hilbert $\mathcal{C}_{0}(X)$-module

$$
\left\{f \in \mathcal{C}\left([0,1], L^{2}(\mathcal{G} \ltimes X)^{\infty}\right) \mid f(0) \in L^{2}(\mathcal{G} \ltimes A)^{\infty}\right\}
$$

with the restriction of $\bar{U}$. This is indeed a cycle for $\operatorname{RK}_{\mathcal{G}, Y}^{0}([0,1] \times X)$ because $\bar{U}$ is invertible on the closure of $X \backslash A$.

The proof that $\operatorname{RK}_{\mathcal{G}, Y}^{*}(X) \cong \lim _{A \in I_{Y}} \operatorname{RK}_{\mathcal{G}, Y}^{*}(A)$ is now finished easily. Since $\bar{A}$ is $Y$-compact for all $A \in I_{Y}$, Lemma 4.17 and the excision property of $\mathrm{RK}_{\mathcal{G}}^{*}$ yield

$$
\operatorname{RK}_{\mathcal{G}, Y}^{*}(A) \cong \operatorname{RK}_{\mathcal{G}}^{*}(\bar{A}, \bar{A} \backslash A) \cong \operatorname{RK}_{\mathcal{G}}^{*}(X, X \backslash A)
$$




\section{Equivariant VECTOR BUNDLES AND THE CROSSED PRODUCT}

We are going to relate $\mathcal{G}$-equivariant vector bundles over $X$ to the $\sigma$ - $C^{*}$-algebra $\sigma-C^{*}(\mathcal{G} \ltimes X)$. As before, $\mathcal{G}$ is a second countable, locally compact, Hausdorff groupoid with Haar system and $X$ is a second countable, locally compact, proper $\mathcal{G}$-space.

We fix an increasing sequence $\left(X_{n}\right)_{n \in \mathbb{N}}$ of $\mathcal{G}$-compact $\mathcal{G}$-invariant subsets with $\bigcup X_{n}=X$ and abbreviate

$$
B_{n}:=C^{*}\left(\mathcal{G} \ltimes X_{n}\right) \cong \mathcal{G} \ltimes \mathcal{C}_{0}\left(X_{n}\right), \quad B:=\lim _{\longleftarrow} B_{n}=\sigma-C^{*}\left(\mathcal{G} \ltimes X_{n}\right) .
$$

Recall that a Hilbert module over $B$ is of the form $\mathcal{E}=\lim \mathcal{E}_{n}$, where each $\mathcal{E}_{n}$ is a Hilbert module over $B_{n}$ and we form the limit with respect to $B_{n+1}$-linear projections $\mathcal{E}_{n+1} \rightarrow \mathcal{E}_{n}$ that induce unitary operators $\mathcal{E}_{n+1} \otimes_{B_{n+1}} B_{n} \cong \mathcal{E}_{n}$.

The $\sigma$ - $C^{*}$-algebras of adjointable and compact operators on $\mathcal{E}$ are

$$
\mathbb{B}(\mathcal{E})=\lim _{\longleftarrow} \mathbb{B}\left(\mathcal{E}_{n}\right) \quad \text { and } \quad \mathbb{K}(\mathcal{E})=\lim _{\longleftarrow} \mathbb{K}\left(\mathcal{E}_{n}\right)
$$

respectively, where the inverse limits are taken in the category of $\sigma$ - $C^{*}$-algebras and hence contain unbounded elements. Let $\mathbb{B}_{\mathrm{b}}(\mathcal{E}) \subseteq \mathbb{B}(\mathcal{E})$ and $\mathbb{K}_{\mathrm{b}}(\mathcal{E}) \subseteq \mathbb{K}(\mathcal{E})$ be the $C^{*}$-algebras of bounded elements, that is, elements $\left(T_{n}\right)_{n \in \mathbb{N}}$ with sup $\left\|T_{n}\right\|<\infty$.

Let $\mathfrak{C}_{B}$ be the category of Hilbert modules over $B$ with bounded adjointable operators as morphisms. Let $\mathfrak{C}_{\mathcal{G}, X}$ be the category of $\mathcal{G}$-equivariant Hilbert modules over $\mathcal{C}_{0}(X)$, with $\mathcal{G}$-equivariant adjointable operators as morphisms. Both $\mathfrak{C}_{B}$ and $\mathfrak{C}_{\mathcal{G}, X}$ are evidently $C^{*}$-categories.

Notation 5.1. Let $B_{\mathbb{K}}$ for a $\sigma-C^{*}$-algebra $B=\lim _{\longleftarrow} B_{n}$ be its stabilisation

$$
B_{\mathbb{K}}:=B \otimes \mathbb{K}\left(\ell^{2} \mathbb{N}\right):=\lim _{\longleftarrow} B_{n} \otimes \mathbb{K}\left(\ell^{2} \mathbb{N}\right) .
$$

Theorem 5.2. The $C^{*}$-categories $\mathfrak{C}_{B}$ and $\mathfrak{C}_{\mathcal{G}, X}$ are equivalent.

$A \mathcal{G}$-equivariant Hilbert $\mathcal{C}_{0}(X)$-module is the space of $\mathcal{C}_{0}$-sections of a $\mathcal{G}$-equivariant Hermitian vector bundle over $X$ if and only if the associated Hilbert $B$-module $\mathcal{E}$ satisfies the following equivalent conditions:

(1) $\mathbb{K}_{\mathrm{b}}(\mathcal{E})=\mathbb{B}_{\mathrm{b}}(\mathcal{E})$;

(2) $\mathbb{K}(\mathcal{E})=\mathbb{B}(\mathcal{E})$;

(3) $\operatorname{id}_{\mathcal{E}} \in \mathbb{K}_{\mathrm{b}}(\mathcal{E})$;

(4) $\operatorname{id}_{\mathcal{E}} \in \mathbb{K}(\mathcal{E})$;

(5) there is a projection $p \in B_{\mathbb{K}}$ whose range is isomorphic to $\mathcal{E}$.

Proof. The first assertion is implicit in the proof of [12, Lemma 20] (in the group case). A $\mathcal{G}$-equivariant Hilbert $\mathcal{C}_{0}(X)$-module $\mathcal{F}$ restricts to $\mathcal{G}$-equivariant Hilbert $\mathcal{C}_{0}\left(X_{n}\right)$-modules $\mathcal{F}_{n}$ for all $n \in \mathbb{N}$. Since $X_{n}$ is $\mathcal{G}$-compact, these correspond to Hilbert $B_{n}$-modules $\mathcal{E}_{n}$ by the construction before [34, Proposition 6.24], and this passage identifies $\mathcal{G}$-equivariant adjointable operators on $\mathcal{F}_{n}$ and adjointable operators on $\mathcal{E}_{n}$. Moreover, compact operators on $\mathcal{E}_{n}$ correspond to $\mathcal{G}$-equivariant adjointable operators $T: \mathcal{F}_{n} \rightarrow \mathcal{F}_{n}$ with $M_{f} T \in \mathbb{K}\left(\mathcal{F}_{n}\right)$ for all $f \in \mathcal{C}_{0}\left(X_{n}\right)$, by 34. Proposition 6.24].

Now we form the Hilbert $B$-module $\mathcal{E}:=\lim \mathcal{E}_{n}$. An adjointable operator on $\mathcal{E}$ restricts to adjointable operators on $\mathcal{E}_{n}$ for all $n \in \mathbb{N}$, and, conversely, a bounded, compatible sequence of adjointable operators on $\mathcal{E}_{n}$ must come from an adjointable operator on $\mathcal{E}$. This reduces the isomorphism $\mathbb{B}_{\mathrm{b}}(\mathcal{E}) \cong \mathbb{B}(\mathcal{F})^{\mathcal{G}}$ to the already know isomorphisms $\mathbb{B}\left(\mathcal{E}_{n}\right) \cong \mathbb{B}\left(\mathcal{F}_{n}\right)^{\mathcal{G}}$ and yields the asserted equivalence of categories.

We can also describe the compact operators:

$$
\begin{aligned}
\mathbb{K}_{\mathrm{b}}(\mathcal{E}) & \cong\left\{T \in \mathbb{B}(\mathcal{F})^{\mathcal{G}}|\varphi(f) T|_{X_{n}} \in \mathbb{K}\left(\mathcal{F}_{n}\right) \text { for all } n \in \mathbb{N}, f \in \mathcal{C}_{0}\left(X_{n}\right)\right\} \\
& \cong\left\{T \in \mathbb{B}(\mathcal{F})^{\mathcal{G}} \mid M_{f} T \in \mathbb{K}(\mathcal{F}) \text { for all } f \in \mathcal{C}_{0}(X)\right\} .
\end{aligned}
$$


The Hilbert module $\mathcal{F}$ is the space of $\mathcal{C}_{0}$-sections of a vector bundle if and only if $M_{f} \in \mathbb{K}(\mathcal{F})$ for all $f \in \mathcal{C}_{0}(X)$; here we may disregard the $\mathcal{G}$-action. For the proof, write $\mathcal{F}$ as a direct summand of $\mathcal{C}_{0}(X)^{\infty}$ and let $p$ be the projection onto $\mathcal{F}$. Since $M_{f} p \in \mathbb{K}\left(\mathcal{C}_{0}(X)^{\infty}\right) \cong \mathcal{C}_{0}(X, \mathbb{K})$, this must be a norm-continuous map from $X$ to the space of projections in $\mathbb{K}:=\mathbb{K}\left(\ell^{2} \mathbb{N}\right)$. Norm continuity implies that the rank of the projection $p$ is locally constant, so that the range of $p$ is a vector bundle.

As a result, $\mathcal{E}$ corresponds to the space of $\mathcal{C}_{0}$-sections of a $\mathcal{G}$-equivariant vector bundle if and only if $\operatorname{id}_{\mathcal{E}} \in \mathbb{K}_{\mathrm{b}}(\mathcal{E})$. It remains to check that the five conditions on $\mathcal{E}$ in the theorem are indeed equivalent. Since $\operatorname{id}_{\mathcal{E}}$ is bounded, $\operatorname{id}_{\mathcal{E}} \in \mathbb{K}_{\mathrm{b}}(\mathcal{E})$ is equivalent to $\operatorname{id}_{\mathcal{E}} \in \mathbb{K}(\mathcal{E})$. Since $\mathbb{K}_{\mathrm{b}}(\mathcal{E})$ and $\mathbb{K}(\mathcal{E})$ are ideals in $\mathbb{B}_{\mathrm{b}}(\mathcal{E})$ and $\mathbb{B}(\mathcal{E})$, respectively, these are equivalent to $\mathbb{K}_{\mathrm{b}}(\mathcal{E})=\mathbb{B}_{\mathrm{b}}(\mathcal{E})$ and $\mathbb{K}(\mathcal{E})=\mathbb{B}(\mathcal{E})$.

By the Stabilisation Theorem for Hilbert modules over $\sigma$ - $C^{*}$-algebras, which is proved like its $C^{*}$-algebra counterpart (see [22]), for any Hilbert module $\mathcal{E}$ over $B$ there is an adjointable isometry $S: \mathcal{E} \rightarrow B^{\infty}:=B \otimes \ell^{2}(\mathbb{N})$. Thus $\mathcal{E}$ is isomorphic to the range of the projection $p:=S S^{*}$. If $\mathrm{id}_{\mathcal{E}}$ is compact, then so is $S=S \circ \mathrm{id}_{\mathcal{E}}$ and hence $p=S \circ S^{*}$. Conversely, if $p$ is compact, so is $S=p \circ S$ and hence $\operatorname{id}_{\mathcal{E}}=S^{*} \circ S$. Hence all five conditions on $\mathcal{E}$ in the statement of the theorem are equivalent as asserted.

Corollary 5.3. The monoid of isomorphism classes of $\mathcal{G}$-equivariant (Hermitian) vector bundles on $X$ is isomorphic to the monoid of idempotents (or projections) in the stable $\sigma-C^{*}$-algebra $B_{\mathbb{K}}:=\sigma-C^{*}(\mathcal{G} \ltimes X)_{\mathbb{K}}$.

Proof. Theorem 5.2 shows that the monoid of isomorphism classes of Hermitian $\mathcal{G}$-equivariant vector bundles on $X$ is equivalent to the monoid of equivalence classes of projections in $B_{\mathbb{K}}$.

We claim that any $\mathcal{G}$-equivariant vector bundle $V$ carries a $\mathcal{G}$-equivariant Hermitian structure. Its construction uses three ingredients. First, we need a Hermitian structure $(\cdot, \cdot)$ on $V$ that need not be $\mathcal{G}$-equivariant - this exists because $X$ is second countable and locally compact, hence paracompact. Secondly, we need a Haar system on $\mathcal{G}$, that is, a left invariant continuous family $\left(\mu_{z}\right)_{z \in Z}$ of non-negative measures on the fibres of the range map $\mathcal{G} \rightarrow Z$. Thirdly, we need a cut-off function, that is, a function $\varphi: X \rightarrow[0, \infty)$ with $\mathcal{G}$-compact support such that $\int \varphi(g \cdot x) \mathrm{d} \mu(g)=1$ for all $x \in X$ - this exists because $\mathcal{G}$ acts properly and $\mathcal{G} \backslash X$ is paracompact. We define

$$
\langle\xi, \eta\rangle:=\int \varphi\left(g^{-1} x\right)\left(g^{-1} \xi, g^{-1} \eta\right) \mathrm{d} \mu(g) \quad \text { for } x \in X, \xi, \eta \in V_{x} .
$$

This is the desired $\mathcal{G}$-invariant Hermitian inner product.

Furthermore, any two $\mathcal{G}$-invariant Hermitian inner products on $V$ are homotopic by an affine homotopy. Hence it makes no difference whether we study vector bundles or Hermitian vector bundles. Moreover, any idempotent in a $\sigma-C^{*}$-algebra is equivalent to a projection, that is, a self-adjoint idempotent, so that it makes no difference whether we use idempotents or projections.

Since projections are automatically bounded, we may replace $B_{\mathbb{K}}$ with its $C^{*}$-subalgebra of bounded elements in Corollary 5.3. But there are good reasons to work with $\sigma-C^{*}$ algebras in this context, such as the non-separability of $\left(B_{\mathbb{K}}\right)_{\mathrm{b}}$ and the topology on unitary groups (see [29, Example 1.2]).

Now we can express $\operatorname{VK}_{\mathcal{G}}^{0}(X)$ and, more generally, the relative theory $\operatorname{VK}_{\mathcal{G}}^{0}(X, A)$, in terms of crossed products:

Definition 5.4. Let $B$ be a $\sigma-C^{*}$-algebra. We let $\operatorname{Vect}(B)$ be the set of Murrayvon Neumann equivalence classes of projections in the stabilisation $B_{\mathbb{K}}$. This is 
a monoid with respect to the orthogonal direct sum, where we use a canonical isomorphism $\mathbb{M}_{2}\left(B_{\mathbb{K}}\right) \cong B_{\mathbb{K}}$. We let $\mathrm{K}_{00}(B)$ the Grothendieck group of $\operatorname{Vect}(B)$.

Definition 5.5. Let $B$ be a $\sigma$ - $C^{*}$-algebra, $J \subseteq B$ a closed ${ }^{*}$-ideal, and $\pi: B_{\mathbb{K}} \rightarrow$ $(B / J)_{\mathbb{K}}$ the quotient map. Consider triples $\left(p^{+}, p^{-}, v\right)$ consisting of two projections $p^{ \pm} \in B_{\mathbb{K}}$ and a partial isometry $v \in(B / J)_{\mathbb{K}}$ whose range and source projections are $\pi\left(p^{-}\right)$and $\pi\left(p^{+}\right)$, respectively, that is, $v v^{*}=\pi\left(p^{-}\right), v^{*} v=\pi\left(p^{+}\right)$. Two such triples $\left(p_{t}^{+}, p_{t}^{-}, v_{t}\right)$ for $t=0,1$ are considered equivalent if there are partial isometries $s^{+}, s^{-}$in $B_{\mathbb{K}}$ with

$$
s^{ \pm}\left(s^{ \pm}\right)^{*}=p_{1}^{ \pm}, \quad\left(s^{ \pm}\right)^{*} s^{ \pm}=p_{0}^{ \pm}, \quad \pi\left(s^{-}\right) v_{0}=v_{1} \pi\left(s^{+}\right) .
$$

The direct sum of such triples is defined as usual and turns the set of equivalence classes of triples into a monoid, which we denote by $\operatorname{Vect}(B, B / J)$.

Call a triple $\left(p^{+}, p^{-}, v\right)$ degenerate if there is a partial isometry $\hat{v} \in B_{\mathbb{K}}$ with $\pi(\hat{v})=v, \hat{v} \hat{v}^{*}=p^{-}$, and $\hat{v}^{*} \hat{v}=p^{+}$. The degenerate triples form a submonoid in $\operatorname{Vect}(B, B / J)$. Let $\mathrm{K}_{00}(B, B / J)$ be the quotient of $\operatorname{Vect}(B, B / J)$ by addition of degenerate cycles.

It is easy to see that $\mathrm{K}_{00}(B, B / J)$ is always a group. The unit element is the class of the degenerate triples, the inverse of $\left(p^{+}, p^{-}, v\right)$ is $\left(p^{-}, p^{+}, v^{*}\right)$.

Theorem 5.6. Let $X$ be a proper $\mathcal{G}$-space and let $A \subseteq X$ be a closed $\mathcal{G}$-invariant subset. Define

$$
B:=\sigma-C^{*}(\mathcal{G} \ltimes X), \quad J:=\lim \mathcal{G} \ltimes \mathcal{C}_{0}\left(X_{n} \backslash A\right) .
$$

Then $J$ is an ideal in $B$. There are canonical monoid isomorphisms

$$
\operatorname{Vect}_{\mathcal{G}}(X) \cong \operatorname{Vect}(B), \quad \operatorname{Vect}_{\mathcal{G}}(X, A) \cong \operatorname{Vect}(B, B / J),
$$

which induce group isomorphisms

$$
\mathrm{VK}_{\mathcal{G}}^{0}(X) \cong \mathrm{K}_{00}(B), \quad \operatorname{VK}_{\mathcal{G}}^{0}(X, A) \cong \mathrm{K}_{00}(B, B / J)
$$

Proof. This follows from Theorem 5.2 and Definitions 2.6, 2.7, 5.4, and 5.5,

5.1. Comparison with representable K-theory. With the same notation as in Theorem 5.6. Corollaries 4.4 and 4.5 yield

$$
\mathrm{RK}_{\mathcal{G}}^{0}(X) \cong \mathrm{K}_{0}(B), \quad \mathrm{RK}_{\mathcal{G}}^{0}(X, A) \cong \mathrm{K}_{0}(J) .
$$

Thus it remains to compare the "naïve" K-theory groups $\mathrm{K}_{00}(B)$ and $\mathrm{K}_{00}(B, B / J)$ with the correct ones. It is easy to construct natural transformations

$$
\begin{aligned}
\mathrm{VK}_{\mathcal{G}}^{0}(X) \cong \mathrm{K}_{00}(B) & \rightarrow \mathrm{K}_{0}(B) \cong \mathrm{RK}_{\mathcal{G}}^{0}(X), \\
\mathrm{VK}_{\mathcal{G}}^{0}(X, A) \cong \mathrm{K}_{00}(B, B / J) & \rightarrow \mathrm{K}_{0}(J) \cong \mathrm{RK}_{\mathcal{G}}^{0}(X, A) .
\end{aligned}
$$

We briefly describe how the map $\operatorname{VK}_{\mathcal{G}}^{0}(X, A) \rightarrow \mathrm{RK}_{\mathcal{G}}^{0}(X, A)$ acts on cycles for the original definitions. Let $\left(V^{+}, V^{-}, \varphi\right)$ be a cycle for $\operatorname{VK}_{\mathcal{G}}^{0}(X, A)$, that is, $V^{+}$and $V^{-}$ are $\mathcal{G}$-equivariant vector bundles on $X$ and $\varphi$ is an isomorphism $\left.\left.V^{+}\right|_{A} \cong V^{-}\right|_{A}$. We equip $V^{ \pm}$with $\mathcal{G}$-invariant inner products, so that their spaces of $\mathcal{C}_{0}$-sections form $\mathcal{G}$-equivariant Hilbert modules over $\mathcal{C}_{0}(X)$.

We extend $\varphi$ to a $\mathcal{G}$-equivariant, contractive vector bundle map $\bar{\varphi}: V^{+} \rightarrow V^{-}$: first use the Tietze Extension Theorem to get a non-equivariant extension; then use the properness of the action to replace this by a $\mathcal{G}$-equivariant function, as in the definition of $T^{\mathcal{G}}$ before Proposition 6.24 in [34]. Since $\bar{\varphi}$ is invertible on $A$, it remains invertible in a neighbourhood of $A$. Using a partition of unity, we can adjust the Hermitian structure so that $\bar{\varphi}$ is unitary in a neighbourhood of $A$. 
Hence we may also define $\operatorname{VK}_{\mathcal{G}}^{0}(X, A)$ using triples $\left(V^{+}, V^{-}, \varphi\right)$, where $V^{+}$and $V^{-}$ are $\mathcal{G}$-equivariant Hermitian vector bundles on $X$ and $\varphi: V^{+} \rightarrow V^{-}$is a $\mathcal{G}$-equivariant operator with $\|\varphi\| \leq 1$ that is unitary in a $\mathcal{G}$-invariant neighbourhood of $A$.

Let $\left(V^{+}, V^{-}, \varphi\right)$ be a cycle for $\operatorname{VK}_{\mathcal{G}}^{0}(X, A)$ of this new kind. Then the spaces $\Gamma_{0}\left(X \backslash A, V^{+}\right)$and $\Gamma_{0}\left(X \backslash A, V^{-}\right)$of $\mathcal{C}_{0^{-}}$-sections are $\mathcal{G}$-equivariant Hilbert modules over $\mathcal{C}_{0}(X \backslash A)$, and $\varphi$ defines an adjointable operator $M_{\varphi}$ between them. The triple

$$
\left(\Gamma_{0}\left(X \backslash A, V^{+}\right), \Gamma_{0}\left(X \backslash A, V^{-}\right), M_{\varphi}\right)
$$

is a cycle for $\operatorname{RK}_{\mathcal{G}, X}^{0}(X \backslash A):=\mathrm{KK}^{\mathcal{G} \ltimes X}\left(\mathcal{C}_{0}(X), \mathcal{C}_{0}(X \backslash A)\right)$ because the functions $\operatorname{id}_{V^{+}}-\varphi^{*} \varphi$ and $\operatorname{id}_{V^{-}}-\varphi \varphi^{*}$ vanish outside an $X$-compact subset of $X \backslash A$. Notice that a subset of $X \backslash A$ is $X$-compact if and only if it is closed.

To get a map $X \backslash A \rightarrow \mathcal{F}_{\mathcal{G}}$ from $\left(V^{+}, V^{-}, \varphi\right)$, we add the degenerate cycle corresponding to the identity operator on $L^{2}(\mathcal{G} \ltimes X \backslash A)^{\infty}$ and use the Equivariant Stabilisation Theorem

$$
\Gamma_{0}\left(X \backslash A, V^{+}\right) \oplus L^{2}(\mathcal{G} \ltimes X \backslash A)^{\infty} \cong L^{2}(\mathcal{G} \ltimes X \backslash A)^{\infty} .
$$

The resulting map $U: X \backslash A \rightarrow \mathcal{F}_{\mathcal{G}}$ is unitary where $\varphi$ is unitary, so that its support is still $X$-compact.

The natural transformation $\operatorname{VK}_{\mathcal{G}}^{0}(X, A) \rightarrow \operatorname{RK}_{\mathcal{G}}^{0}(X, A)$ also yields one from $\operatorname{VK}_{\mathcal{G}}^{-n}(X, A)$ to $\operatorname{RK}_{\mathcal{G}}^{-n}(X, A)$ for all $n \in \mathbb{N}$, using (2.8) and the natural isomorphisms

(5.7) $\operatorname{RK}_{\mathcal{G}}^{-n}(X, A) \cong \operatorname{RK}_{\mathcal{G}}^{0}\left(X \times \mathbb{R}^{n}, A \times \mathbb{R}^{n}\right) \cong \operatorname{RK}_{\mathcal{G}}^{0}\left(X \times \mathbb{S}^{n}, X \times\{1\} \cup A \times \mathbb{S}^{n}\right)$,

where we use the excision property (4.15).

Now we have constructed a natural transformation $\operatorname{VK}_{\mathcal{G}}^{*}(X, A) \rightarrow \operatorname{RK}_{\mathcal{G}}^{*}(X, A)$. This is not an isomorphism in general, as shown by several counterexamples [19,28, 32. The following counterexample by Juliane Sauer is particularly simple.

Example 5.8 (see [32]). Consider the compact group $K:=\prod_{n \in \mathbb{Z}} \mathbb{Z} / 2$ and let $\alpha$ be the automorphism on $K$ that shifts the copies of $\mathbb{Z} / 2$ to the left. Form the semidirect product group $G:=\mathbb{Z} \ltimes_{\alpha} K$; this is a totally disconnected group. Let $K$ act trivially and $\mathbb{Z}$ by translation on $\mathbb{R}$. This combines to a proper, cocompact action of $G$ on $\mathbb{R}$.

It is shown in 32 that the map

$$
\mathrm{VK}_{G}^{1}(\mathbb{R}, \mathbb{Z}) \rightarrow \mathrm{RK}_{G}^{1}(\mathbb{R}, \mathbb{Z})
$$

is not surjective. The idea is the following. On the right hand side, we compute $\operatorname{RK}_{G}^{1}(\mathbb{R}, \mathbb{Z}) \cong \mathrm{K}_{G}^{1}(\mathbb{R}, \mathbb{Z}) \cong \mathrm{K}^{1}\left(G \ltimes \mathcal{C}_{0}(\mathbb{R} \backslash \mathbb{Z})\right) \cong \mathrm{K}^{1}\left(\mathcal{C}_{0}((0,1)) \otimes C^{*}(K)\right) \cong \operatorname{Rep}(K)$.

But any $G$-equivariant vector bundle on $\mathbb{R} \times \mathbb{S}^{1}$ must carry the trivial representation of $K$ because this is the only representation that is fixed by the automorphism $\alpha$. Hence $\mathrm{VK}_{G}^{1}(\mathbb{R}, \mathbb{Z})=\mathrm{VK}_{\mathbb{Z}}^{1}(\mathbb{R}, \mathbb{Z}) \cong \mathbb{Z}$ is much smaller than $\mathrm{RK}_{G}^{1}(\mathbb{R}, \mathbb{Z})$.

A similar computation shows that the map $\mathrm{VK}_{G}^{0}\left(\mathbb{R} \times \mathbb{S}^{1}\right) \rightarrow \mathrm{RK}_{G}^{0}\left(\mathbb{R} \times \mathbb{S}^{1}\right)$ is not surjective, providing a counterexample for the absolute theories.

By the way, since $\mathbb{Z}$ acts freely and properly on $\mathbb{R}$, the crossed product groupoid $G \ltimes \mathbb{R}$ is Morita equivalent to a locally trivial bundle of compact groups on $\mathbb{S}^{1}=\mathbb{R} / \mathbb{Z}$ whose fibre is $K$ everywhere. More precisely, we consider the trivial bundle of groups $[0,1] \times K$ and identify $(0, g) \sim(1, \alpha(g))$ for all $g \in K$ to get a locally trivial bundle on $\mathbb{S}^{1}$. Since all our theories are evidently Morita invariant, we also get counterexamples for this groupoid, which is a locally trivial bundle of compact groups with compact base.

Several authors have established $\operatorname{VK}_{G}^{*}(X, A) \cong \mathrm{RK}_{G}^{*}(X, A)$ for some classes of cocompact group actions. Of course, if $X$ is $G$-compact then we can replace 
$\operatorname{RK}_{G}^{*}(X, A)$ by $\mathrm{K}_{G}^{*}(X, A) \cong \mathrm{K}_{G}^{*}(X \backslash A)$. If $G$ is almost connected or a matrix group, then Chris Phillips ([27]) proves an isomorphism $\operatorname{VK}_{G}^{*}(X, A) \cong \mathrm{K}_{G}^{*}(X \backslash A)$ for all $G$-compact proper $G$-spaces $X$. Wolfgang Lück and Bob Oliver ([19]) show this if $G$ is discrete and $(X, A)$ is a finite $G$-CW-pair. The latter result is extended by Juliane Sauer (32] to totally disconnected groups that are projective limits of discrete groups. These results are contained in Theorem 6.15 below.

Even without a group action, there are non-compact spaces for which vector bundles do not generate the representable K-theory. It is known to experts that $\mathrm{VK}^{*}(X) \cong \mathrm{RK}^{*}(X)$ if $X$ is, say, a finite-dimensional CW-complex; more generally, this holds for paracompact Hausdorff spaces with finite covering dimension. But we could not find a proof of this in the literature. We do not consider equivariant versions of this result here.

\section{Approximate units of projections}

Definition 6.1. A (separable) $\sigma-C^{*}$-algebra $B$ has an approximate unit of projections if there is a sequence of projections $\left(p_{n}\right)$ in $B$ with $\lim _{n \rightarrow \infty} p_{n} \cdot b \cdot p_{n}=b$ for all $b \in B$.

Since a sequence in $B=\lim B_{n}$ converges if and only if its image in $B_{n}$ converges for all $n \in \mathbb{N}$, a sequence of projections $\left(p_{n}\right)$ in $B=\lim B_{n}$ is an approximate unit in $B$ if and only if its image in $B_{n}$ is an approximate unit for each $n \in \mathbb{N}$. But it does not suffice merely to assume that all $B_{n}$ have approximate units of projections because it is not clear whether projections in $B_{n}$ lift to projections in $B$.

If $B$ has an approximate unit of projections, so has $B_{\mathbb{K}}$ : simply consider the sequence $\left(p_{n} \otimes q_{n}\right)$ where $\left(p_{n}\right)$ and $\left(q_{n}\right)$ are approximate units of projections in $B$ and $\mathbb{K}$, respectively. The following example shows that the converse need not hold:

Example 6.2. We describe a $C^{*}$-algebra $B$ without an approximate unit of projections for which $B_{\mathbb{K}}$ has one. Let

$$
B:=\left\{\left(\begin{array}{ll}
a & b \\
c & d
\end{array}\right) \in \mathbb{M}_{2} \otimes \mathcal{C}([0,1]) \mid b(0)=c(0)=d(0)=0\right\} .
$$

This is the $C^{*}$-algebra of a proper groupoid, namely, the groupoid associated to the equivalence relation on

$$
[0,1] \sqcup(0,1]=[0,1] \times\{0\} \cup(0,1] \times\{1\}
$$

that identifies $(t, 0) \sim(t, 1)$ for all $t \in(0,1]$. Of course, this groupoid is Morita equivalent to $[0,1]$ with only identity morphisms.

Correspondingly, $B$ is Morita equivalent to $C([0,1])$. Thus $B_{\mathbb{K}} \cong C([0,1])_{\mathbb{K}}$. This $C^{*}$-algebra has an approximate unit of projections because $C([0,1])$ is unital. But if $p \in B$ is a projection, then $p(0)$ must have rank 1 , so that $p$ has rank 1 on all of $[0,1]$. Hence $B$ itself contains no approximate unit of projections.

Since our notions should be Morita invariant, it is better to require only an approximate unit of projections in $B_{\mathbb{K}}$, not in $B$.

A local Banach algebra $A$ for which $\mathbb{M}_{\infty}(A)$ has an approximate unit of projections is called stably unital in [4, Definition 5.5.4]. We avoid this name for two reasons: first, $\sigma-C^{*}$-algebras are local Banach algebras if and only if they are $C^{*}$-algebras; secondly, we also consider $\sigma$ - $C^{*}$-algebras that are stably isomorphic to unital $\sigma-C^{*}$-algebras, and these also deserve to be called stably unital.

Proposition 6.3. Let $B$ be a $C^{*}$-algebra for which $B_{\mathbb{K}}$ has an approximate unit of projections. Then $\mathrm{K}_{00}(B) \cong \mathrm{K}_{0}(B)$. If $J \subseteq B$ is a closed ideal, then $\mathrm{K}_{00}(B, B / J) \cong$ $\mathrm{K}_{0}(J)$. 
Proof. The first assertion is [4, Proposition 5.5.5]. The second result is well-known if $B$ is unital (see [4, Theorem 5.4.2]). We explain how to reduce the general case to this special case.

Stabilising $J$ and $B$, we may assume that $B$ itself has an approximate unit of projections $\left(p_{n}\right)_{n \in \mathbb{N}}$. The extension $J \longmapsto B \rightarrow B / J$ is the inductive limit in the category of $C^{*}$-algebras of the extensions

$$
p_{n} J p_{n} \longmapsto p_{n} B p_{n} \rightarrow \pi\left(p_{n}\right)(B / J) \pi\left(p_{n}\right) .
$$

Since $K_{0}$ is continuous for such inductive limits, and since the same holds for our relative theory $\mathrm{K}_{00}(B, B / J)$, it suffices to prove

$$
\mathrm{K}_{0}\left(p_{n} J p_{n}\right)=\mathrm{K}_{00}\left(p_{n} B p_{n}, \pi\left(p_{n}\right)(B / J) \pi\left(p_{n}\right)\right)
$$

for all $n \in \mathbb{N}$. This reduces the assertion to the known case where $B$ is unital.

Proposition 6.3 fails for $\sigma$ - $C^{*}$-algebras, in general. The main source of problems is that the set of invertible elements in a $\sigma$ - $C^{*}$-algebra is not open.

Theorem 6.4. Let $\mathcal{G}$ be a second countable, locally compact, Hausdorff groupoid with Haar system and let $X$ be a proper, $\mathcal{G}$-compact, second countable $\mathcal{G}$-space. Suppose that $C^{*}(\mathcal{G} \ltimes X)_{\mathbb{K}}$ has an approximate unit of projections.

Then the canonical map

$$
\operatorname{VK}_{\mathcal{G}}^{*}(X, A) \rightarrow \operatorname{RK}_{\mathcal{G}}^{*}(X, A) \cong \mathrm{K}_{\mathcal{G}}^{*}(X, A) \cong \mathrm{K}_{\mathcal{G}}^{*}(X \backslash A)
$$

is an isomorphism for all closed $\mathcal{G}$-invariant subsets $A \subseteq X$.

Let $Y$ be a $\mathcal{G} \ltimes X$-space and let $S_{X}$ be the directed set of closed, $\mathcal{G}$-invariant, $X$-compact subsets of $Y$. For $A \in S_{X}$, let $\partial A$ be the boundary of $A$ as a subset of $Y$. Then

$$
\operatorname{RK}_{\mathcal{G}, X}^{*}(Y) \cong \lim _{A \in S_{X}} \operatorname{VK}_{\mathcal{G}}^{*}(A, \partial A) .
$$

Proof. Define $B$ and $J$ as in Theorem 5.6. Thus

$$
\mathrm{VK}_{\mathcal{G}}^{0}(X) \cong \mathrm{K}_{00}(B), \quad \operatorname{VK}_{\mathcal{G}}^{0}(X, A) \cong \mathrm{K}_{00}(B, B / J) \text {. }
$$

Corollary 4.4 yields $\operatorname{RK}_{\mathcal{G}}^{0}(X) \cong \mathrm{K}_{0}(B)$, and Corollary 4.5 and the definition of the relative theory in (4.14) yield $\operatorname{RK}_{\mathcal{G}}^{0}(X, A) \cong \mathrm{K}_{0}(J)$. Hence the first two assertions of the theorem follow from Proposition 6.3 .

To prove the last assertion, we claim that there are natural isomorphisms

$$
\operatorname{RK}_{\mathcal{G}, X}^{*}(Y) \cong \underset{A \in S_{X}}{\lim _{A}} \operatorname{RK}_{\mathcal{G}}^{*}\left(Y, Y \backslash A^{\circ}\right) \cong \underset{A \in \vec{S}_{X}}{\lim _{\mathcal{G}}} \operatorname{RK}_{\mathcal{G}}^{*}(A, \partial A) \cong \underset{A \in S_{X}}{\lim _{\mathcal{G}}} \operatorname{VK}_{\mathcal{G}}^{*}(A, \partial A) .
$$

Here $A^{\circ}$ denotes the interior of $A$, so that $Y \backslash A^{\circ}$ is closed in $Y$ for all $A \in S_{X}$. The first isomorphism follows from Theorem 4.19. The second isomorphism is the excision isomorphism (4.15) for $\mathrm{RK}_{\mathcal{G}}^{*}$. For the third one, we use that $\mathcal{G} \ltimes \mathcal{C}_{0}(A)_{\mathbb{K}}$ has an approximate unit of projections as well. This is true because the proper map $A \rightarrow X$ induces an essential ${ }^{*}$-homomorphism $\mathcal{G} \ltimes \mathcal{C}_{0}(X)_{\mathbb{K}} \rightarrow \mathcal{G} \ltimes \mathcal{C}_{0}(A)_{\mathbb{K}}$, which maps the approximate unit of projections in $\mathcal{G} \ltimes \mathcal{C}_{0}(X)_{\mathbb{K}}$ to one in $\mathcal{G} \ltimes \mathcal{C}_{0}(A)_{\mathbb{K}}$.

The inductive limit $\lim _{A \in S_{X}} \operatorname{VK}_{\mathcal{G}}^{*}(A, \partial A)$ implicitly uses maps

$$
\operatorname{VK}_{\mathcal{G}}^{*}(A, \partial A) \rightarrow \operatorname{VK}_{\mathcal{G}}^{*}(B, \partial B)
$$

for $A, B \in S_{X}$ with $A \subseteq B$. To construct these, we need non-trivial excision isomorphisms

$$
\mathrm{VK}_{\mathcal{G}}^{*}(A, \partial A) \cong \mathrm{VK}_{\mathcal{G}}^{*}\left(B, B \backslash A^{\circ}\right) \text {. }
$$

We have excision here because both sides are naturally isomorphic to $\operatorname{RK}_{\mathcal{G}}^{*}(A, \partial A)$ and $\operatorname{RK}_{\mathcal{G}}^{*}\left(B, B \backslash A^{\circ}\right)$, respectively, which do satisfy excision by (4.15).

It will be useful for later to formalise an idea in the proof of Theorem 6.4 
Proposition 6.5. Let $f: X \rightarrow Y$ be a $\mathcal{G}$-equivariant continuous map between two proper $\mathcal{G}$-spaces. If $\sigma-C^{*}(\mathcal{G} \ltimes Y)_{\mathbb{K}}$ has an approximate unit of projections, then so has $\sigma-C^{*}(\mathcal{G} \ltimes X)_{\mathbb{K}}$.

In particular, let $\mathcal{E G}$ be a universal proper $\mathcal{G}$-space. If $\sigma-C^{*}(\mathcal{G} \ltimes \mathcal{E} \mathcal{G})_{\mathbb{K}}$ has an approximate unit of projections, then $\sigma-C^{*}(\mathcal{G} \ltimes X)_{\mathbb{K}}$ has an approximate unit of projections for all proper $\mathcal{G}$-spaces $X$.

Proof. Choose exhausting sequences $\left(X_{n}\right)$ and $\left(Y_{n}\right)$ of $\mathcal{G}$-compact subsets as in Theorem 4.2. The map $f: X \rightarrow Y$ restricts to proper maps $X_{n} \rightarrow Y_{n}$, no matter whether $f$ itself is proper. This is because $X_{n}$ and $Y_{n}$ are $\mathcal{G}$-compact and proper, so that any continuous $\mathcal{G}$-equivariant map between them is proper. Hence we get induced *-homomorphisms $\mathcal{G} \ltimes \mathcal{C}_{0}\left(Y_{n}\right) \rightarrow \mathcal{G} \ltimes \mathcal{C}_{0}\left(X_{n}\right)$, which are essential. The resulting ${ }^{*}$-homomorphism

$$
\sigma-C^{*}(\mathcal{G} \ltimes Y)_{\mathbb{K}} \rightarrow \sigma-C^{*}(\mathcal{G} \ltimes X)_{\mathbb{K}}
$$

maps an approximate unit of projections for $Y$ to one for $X$, yielding the first assertion. The last assertion follows because any proper $\mathcal{G}$-space maps to $\mathcal{E} \mathcal{G}$.

6.1. General existence criteria. Theorem 6.4 motivates us to search for approximate units of projections in stable $C^{*}$-algebras and $\sigma$ - $C^{*}$-algebras. We will see that there is an approximate unit of projections in the stabilisation if there are enough projections in a weaker sense. Various counterexamples suggest that we cannot do much better here.

To simplify our notation, we consider only stable $\sigma$ - $C^{*}$-algebras from now on, that is, we assume $B \cong B_{\mathbb{K}}$. This can be achieved by replacing $B$ by $B_{\mathbb{K}}$.

Definition 6.6. Let $\mathcal{P}(B)$ denote the primitive ideal space of a $C^{*}$-algebra $B$, equipped with the hull-kernel topology. For a $\sigma$ - $C^{*}$-algebra $B=\lim B_{n}$, the projections $B_{n+1} \rightarrow B_{n}$ yield an inductive system of closed embeddings of topological spaces $\mathcal{P}\left(B_{n}\right) \rightarrow \mathcal{P}\left(B_{n+1}\right)$. We let $\mathcal{P}(B)$ be the direct limit of this system or, less formally, $\mathcal{P}(B):=\bigcup_{n=1}^{\infty} \mathcal{P}\left(B_{n}\right)$.

For each primitive ideal $\mathfrak{p} \in \mathcal{P}\left(B_{n}\right) \subseteq \mathcal{P}(B)$, we get a $C^{*}$-algebra quotient $B / \mathfrak{p}:=B_{n} / \mathfrak{p}$, which does not depend on the choice of $n$.

Definition 6.7. A $\sigma$ - $C^{*}$-algebra $B=\lim B_{n}$ is called $\sigma$-unital if it contains a strictly positive element $h \in B$.

It is easy to see that $B=\lim _{n} B_{n}$ is $\sigma$-unital if and only if $B_{n}$ is $\sigma$-unital for all $n \in \mathbb{N}$. This holds, for instance, if all $B_{n}$ are separable.

Theorem 6.8. A stable, $\sigma$-unital $\sigma-C^{*}$-algebra $B$ contains an approximate unit of projections if and only if for each primitive ideal $\mathfrak{p}$ in $B$ there is a projection $q$ in $B$ that does not belong to $\mathfrak{p}$.

Proof. Suppose first that $B \cong B_{\mathbb{K}}$ contains an approximate unit of projections $\left(e_{n}\right)_{n \in \mathbb{N}}$. If $\mathfrak{p} \in B$, then the images of $e_{n}$ in $B / \mathfrak{p}$ form an approximate unit of projections as well. Hence $e_{n} \notin \mathfrak{p}$ for some $n \in \mathbb{N}$.

The converse direction is more interesting. For a projection $q \in B$, let $I_{q}$ be the closed *-ideal generated by $q$, that is, the closed linear span of $a q b$ for $a, b \in B$. The ideal $I_{q}$ is Morita equivalent to the corner $q B q$ via the imprimitivity bimodule $q B$ (see [6]). Thus each ideal $I_{q}$ is Morita equivalent to a unital $C^{*}$-algebra. We identify $\mathcal{P}\left(I_{q}\right)$ with an open subset in $\mathcal{P}(B)$ in the usual way. We have $\mathfrak{p} \in \mathcal{P}\left(I_{q}\right)$ if and only if $q \notin \mathfrak{p}$. Hence the subsets $\mathcal{P}\left(I_{q}\right)$ for projections $q \in B$ form an open covering of $\mathcal{P}(B)$. More precisely, they form open coverings of $\mathcal{P}\left(B_{n}\right)$ for each $n \in \mathbb{N}$.

Let $h \in B$ be strictly positive. The subsets

$$
X_{n}:=\left\{\mathfrak{p} \in \mathcal{P}(B) \mid\|h\|_{B / \mathfrak{p}} \geq 1 / n\right\}
$$


for $n \in \mathbb{N}$ have quasi-compact intersection with $\mathcal{P}\left(B_{k}\right)$ for each $k \in \mathbb{N}$. They form an increasing sequence with $\bigcup_{n \in \mathbb{N}} X_{n}=\mathcal{P}(B)$ because $h \in B$ is strictly positive. Let $n \in \mathbb{N}$. Since $X_{n} \cap \mathcal{P}\left(B_{n}\right)$ is quasi-compact, we can find projections $q_{1}, \ldots, q_{j} \in B$ with

$$
X_{n} \cap \mathcal{P}\left(B_{n}\right) \subseteq \mathcal{P}\left(I_{q_{1}}\right) \cup \cdots \cup \mathcal{P}\left(I_{q_{j}}\right) .
$$

The projection $q_{1} \oplus \cdots \oplus q_{j} \in \mathbb{M}_{j}(B)$ is Murray-von Neumann equivalent to a projection $e_{n}$ in $B$ because $B$ is stable. It is easy to see that Murray-von Neumann equivalent projections generate the same ideal. Hence the primitive ideal space of $I_{n}:=I_{e_{n}}$ contains $X_{n} \cap \mathcal{P}\left(B_{n}\right)$. Since $\bigcup X_{n}=\mathcal{P}(B)$, we get $\bigcup \mathcal{P}\left(I_{n}\right)=\mathcal{P}(B)$. This implies that $\bigcup I_{n}$ is dense in $B$ : a subset is dense in $B$ if and only if its image in $B_{k}$ is dense for all $k \in \mathbb{N}$, and open subsets of $\mathcal{P}\left(B_{k}\right)$ are in bijection with ideals in $B_{k}$.

Each of the ideals $I_{n}$ is Morita equivalent to a unital $\sigma$ - $C^{*}$-algebra, namely, $e_{n} B e_{n}$. Therefore, $\left(I_{n}\right)_{\mathbb{K}}$ contains an approximate unit of projections $\left(e_{n, k}\right)_{k \in \mathbb{N}}$. Since $\bigcup\left(I_{n}\right)_{\mathbb{K}}$ is dense in $B_{\mathbb{K}}, e_{n}^{\prime}:=e_{n, k_{n}}$ for a suitable function $n \mapsto k_{n}$ is an approximate unit of projections in $B_{\mathbb{K}}$.

Definition 6.9. A set of projections $S$ is called full if for any primitive ideal $\mathfrak{p}$ in $B$, there is $q \in S$ that does not belong to $\mathfrak{p}$. A single projection $q$ is called full if $\{q\}$ is.

Thus Theorem 6.8 asserts that a $\sigma$-unital stable $\sigma$ - $C^{*}$-algebra has an approximate unit of projections if and only if it contains a full set of projections.

Corollary 6.10. The following are equivalent for a $\sigma$-unital stable $C^{*}$-algebra $B$ :

(1) B contains a full projection;

(2) $B$ is Morita equivalent to a unital algebra;

(3) $B$ contains an approximate unit of projections and $\mathcal{P}(B)$ is quasi-compact;

(4) $B$ contains a full set of projections and $\mathcal{P}(B)$ is quasi-compact.

Statements (1) and (2) remain equivalent for a $\sigma$-unital stable $\sigma$ - $C^{*}$-algebra $B$.

Proof. $(1) \Longleftrightarrow(2)$ for $\sigma$ - $C^{*}$-algebras: If $p \in B$ is a full projection, then the ideal $I_{p}$ generated by $p$ is all of $B$, and Morita equivalent to the unital algebra $p B p$. Conversely, if $B$ is Morita equivalent to a unital $\sigma-C^{*}$-algebra $A$, then $B \cong B_{\mathbb{K}} \cong A_{\mathbb{K}}$, and the class of the unit in $A$ is a full projection in $A_{\mathbb{K}}$.

Now we assume that $B$ is a $C^{*}$-algebra.

$(2) \Longrightarrow(3)$ : since $B$ is stable, $(2)$ implies $B \cong A_{\mathbb{K}}$ for a unital $C^{*}$-algebra $A$. Then $1_{A} \otimes e_{n}$ is an approximate unit of projections in $A$, where $\left(e_{n}\right)$ is one in $\mathbb{K}$, and $\mathcal{P}(A) \cong \mathcal{P}(B)$ is quasi-compact.

$(3) \Longrightarrow(4)$ is trivial.

$(4) \Longrightarrow(1)$ : This follows from the proof of Theorem 6.8. Since $\mathcal{P}(B)$ is quasicompact, we have $\mathcal{P}(B)=\mathcal{P}\left(I_{n}\right)$ for some $n \in \mathbb{N}$. Hence the projection $q_{n}$ that generates $I_{n}$ is full.

Corollary 6.11. Let $B$ be a $\sigma$-unital $C^{*}$-algebra. If $B_{\mathbb{K}}$ contains an approximate unit of projections, then there is an increasing sequence of quasi-compact open subsets $U_{n} \subseteq \mathcal{P}(B)$ with $\bigcup U_{n}=\mathcal{P}(B)$.

Proof. This follows from the proof of Theorem 6.8. The subsets $\mathcal{P}\left(I_{n}\right)$ are open and quasi-compact because each $I_{n}$ is Morita equivalent to a unital $C^{*}$-algebra.

Example 6.12. Consider again the situation of Example 5.8 that is, let $\mathcal{G}$ be (Morita equivalent) to the locally trivial bundle of compact groups on $\mathbb{S}^{1}$ with fibre $K$, twisted by the automorphism $\alpha$. The primitive ideal space $P$ of $C^{*}(\mathcal{G})$ is Hausdorff in this case, and the map $P \rightarrow \mathbb{S}^{1}$ is a covering map with fibre $\mathcal{P}\left(C^{*} K\right)=\widehat{K}$. The 
holonomy of this covering is given by the action of the automorphism $\alpha$ on $\widehat{K}$. More explicitly,

$$
\widehat{K}=\bigoplus_{n \in \mathbb{Z}} \widehat{\mathbb{Z} / 2} \cong \bigoplus_{n \in \mathbb{Z}} \mathbb{Z} / 2
$$

with $\alpha$ acting by translation. This action is free on $\widehat{K} \backslash\{\tau\}$, where $\tau$ denotes the trivial representation. Thus $\mathcal{P}\left(C^{*} \mathcal{G}\right)$ contains several copies of $\mathbb{R}$ - one for each orbit of $\alpha$ on $\widehat{K} \backslash\{\tau\}$ - and one copy of $\mathbb{S}^{1}$. Since $\mathbb{R}$ contains no quasi-compact open subsets, the necessary condition of Corollary 6.11 is violated in this case.

Example 6.13. The necessary condition in Corollary 6.11 is not sufficient, even if we restrict attention to, say, type I $C^{*}$-algebras. Counterexamples come from continuous trace $C^{*}$-algebras (see also 35$]$ ). Let $B$ be a continuous trace $C^{*}$-algebra with connected spectrum $X$. Stable isomorphism classes of continuous trace $C^{*}$-algebras with spectrum $X$ are classified by their Dixmier-Douady invariant in the Cech cohomology $H^{3}(X, \mathbb{Z})$ (see $[9$, Theorem 9.9]). If $p$ is a projection in a stable continuous trace $C^{*}$-algebra $B$ over $X$, then the rank of $p$ is a constant function on $X$. Thus $p B p$ is a locally trivial bundle of finite-dimensional matrix algebras. But this implies that the Dixmier-Douady invariant in $H^{3}(X, \mathbb{Z})$ is torsion (see 9 , Theorem $9.13])$.

Hence a continuous trace $C^{*}$-algebra with non-torsion Dixmier-Douady invariant contains no projections, even stably. In contrast, a continuous trace $C^{*}$-algebra with torsion Dixmier-Douady invariant is Morita equivalent to a unital $C^{*}$-algebra and hence contains an approximate unit of projections in its stabilisation by Corollary 6.10

Example 6.13 shows that the existence of an approximate unit of projections in the stabilisation is a very subtle global question. This explains why our criteria for groupoid $C^{*}$-algebras require the existence of some equivariant vector bundles to begin with.

\subsection{Application to groupoids.}

Theorem 6.14. Let $\mathcal{G}$ be a groupoid and let $X$ be a proper $\mathcal{G}$-space. The $\sigma-C^{*}$ algebra $\sigma-C^{*}(\mathcal{G} \ltimes X)_{\mathbb{K}}$ has an approximate unit of projections if and only if, for each $x \in X$ and each irreducible representation $\varrho: \mathcal{G}_{x}^{x} \rightarrow \mathbb{M}_{n}(\mathbb{C})$ of its stabiliser $\mathcal{G}_{x}^{x}$, there is a $\mathcal{G}$-equivariant vector bundle on $X$ whose fibre at $x$ contains the representation $\varrho$.

The $\sigma$-C $C^{*}$-algebra $\sigma-C^{*}(\mathcal{G} \ltimes X)_{\mathbb{K}}$ is Morita equivalent to a unital $\sigma$ - $C^{*}$-algebra if and only if there is a single $\mathcal{G}$-equivariant vector bundle on $X$ whose fibre at $x$ contains all irreducible representations of $\mathcal{G}_{x}^{x}$ for all $x \in X$.

Proof. First we recall how to compute the primitive ideal space of $\sigma-C^{*}(\mathcal{G} \ltimes X)$. It suffices to do this in the cocompact case where $\sigma-C^{*}(\mathcal{G} \ltimes X)$ is a $C^{*}$-algebra because $\mathcal{P}\left(\sigma-C^{*}(\mathcal{G} \ltimes X)\right)=\bigcup \mathcal{P}\left(C^{*}\left(\mathcal{G} \ltimes X_{n}\right)\right)$; here $X_{n}$ is an increasing sequence of cocompact $\mathcal{G}$-invariant subsets with $\bigcup X_{n}=X$.

Any irreducible ${ }^{*}$-representation of $C^{*}\left(\mathcal{G} \ltimes X_{n}\right)$ is carried by a single orbit because the central subalgebra $\mathcal{C}_{0}\left(\mathcal{G} \backslash X_{n}\right)$ of $\mathcal{M}\left(C^{*}\left(\mathcal{G} \ltimes X_{n}\right)\right)$ must act by a character. The irreducible representations carried by the orbit $\mathcal{G} \cdot x$ correspond bijectively to the irreducible representations of the restricted groupoid $\mathcal{G} \ltimes(\mathcal{G} \cdot x)$, which is Morita equivalent to the stabiliser $\mathcal{G}_{x}^{x}$ of $x$ because it is proper and transitive. Thus irreducible representations correspond bijectively to $\bigsqcup_{x \in R} \widehat{\mathcal{G}_{x}^{x}}$, where $R$ is a set of representatives for the $\mathcal{G}$-orbits in $X$. It follows easily that each irreducible representation of $C^{*}\left(\mathcal{G} \ltimes X_{n}\right)$ is completely continuous. Hence the primitive ideal space agrees with the space of irreducible representations.

Theorem $\left[5.2\right.$ shows that equivalence classes of idempotents in $\sigma-C^{*}(\mathcal{G} \ltimes X)_{\mathbb{K}}$ correspond bijectively to isomorphism classes of $\mathcal{G}$-equivariant vector bundles on $X$. 
The projection associated to a $\mathcal{G}$-equivariant vector bundle $V$ vanishes at a representation $\varrho \in \widehat{\mathcal{G}_{x}^{x}}$ if and only if the fibre $V_{x}$, which is a representation of $\mathcal{G}_{x}^{x}$, does not contain $\varrho$. Hence the assertion follows from Theorem 6.8. Furthermore, a single vector bundle contains all irreducible representations of stabilisers if and only if the corresponding projection in $\sigma-C^{*}(\mathcal{G} \ltimes X)_{\mathbb{K}}$ is full. Such a projection exists if and only if $\sigma-C^{*}(\mathcal{G} \ltimes X)_{\mathbb{K}}$ is Morita equivalent to a unital $\sigma$ - $C^{*}$-algebra.

Theorem 6.14 seems disappointing at first sight because we require the existence of some equivariant vector bundles to begin with. Examples 6.12 and 6.13 suggest that we must construct some equivariant vector bundles by hand.

The following theorem contains the three previously known situations where equivariant K-theory for group actions can be computed by equivariant vector bundles (see [19,27, 32, .

Theorem 6.15. Let $G$ be a locally compact group and let $X$ be a proper $G$-compact $G$-space. The $\sigma-C^{*}$-algebra $\sigma-C^{*}(G \ltimes X)_{\mathbb{K}}$ has an approximate unit of projections in each of the following cases:

(1) if $G$ is a closed subgroup of an almost connected group $H$, that is, the component group $\pi_{0}(H)$ is compact;

(2) if $G$ is discrete, $G \backslash X$ has finite covering dimension, and all finite subgroups of $G$ have order at most $N$ for some $N \in \mathbb{N}$;

(3) more generally, it suffices to assume that there is a decreasing sequence of compact, open, normal subgroups $\left(K_{n}\right)_{n \in \mathbb{N}}$ such that

- $K_{n}=\{1\}$;

- for each $n \in \mathbb{N}$ there is $N_{n} \in \mathbb{N}$ such that all finite subgroups of the discrete group $G / K_{n}$ have at most $N_{n}$ elements;

- the orbit spaces $K_{n} \backslash X$ have finite covering dimension for all $n \in \mathbb{N}$.

Furthermore, in the second case, $\sigma-C^{*}(G \ltimes X)_{\mathbb{K}}$ contains a full projection, so that $\sigma-C^{*}(G \ltimes X)$ is Morita equivalent to a unital $\sigma-C^{*}$-algebra.

Proof. We treat case (1) first. We induce the action of $G$ to an action $H \times_{G} X$ of $H$, which is still cocompact. The groupoids $G \ltimes X$ and $H \ltimes\left(H \times_{G} X\right)$ are Morita equivalent, so that it makes no difference which one we study. Let $K \subseteq H$ be a maximal compact subgroup. Then the quotient space $H / K$ is a universal proper $H$-space by [1]. This model of $\mathcal{E} H$ is $H$-compact, and $C^{*}(H \ltimes H / K)$ is Morita equivalent to $C^{*}(K)$. Since the latter has an approximate unit of projections, Proposition 6.5 yields one in $\sigma-C^{*}\left(H \ltimes\left(H \times_{G} X\right)\right)_{\mathbb{K}}$ and hence in $\sigma-C^{*}(G \ltimes X)_{\mathbb{K}}$.

Now we turn to case (2). For a discrete group $G$, Wolfgang Lück and Bob Oliver construct a full $G$-equivariant vector bundle on any finite-dimensional proper $G$-CW-complex whose isotropy groups have bounded order (see [19, Corollary 2.7]). Proposition 6.5yields our assertion for $X$ if we can find a continuous $G$-map from $X$ to such a space. This is an easy application of partitions of unity.

Any proper group action is locally induced, that is, there is an open $G$-invariant covering $\mathcal{U}$ such that for each $U \in \mathcal{U}$, there is a $G$-equivariant homeomorphism $U \cong G \times_{H} Y$ for some finite subgroup $H \subseteq G$ and some $H$-space $Y$ (see [2,7]). Equivalently, there is a $G$-equivariant map $U \rightarrow G / H$. Since the orbit space $G \backslash X$ has finite covering dimension, we may assume that $\mathcal{U}$ splits into $n+1$ subsets $\mathcal{U}_{0}, \ldots, \mathcal{U}_{n}$ such that $U \cap V=\emptyset$ for all $U, V \in \mathcal{U}_{j}$ for the same $j$. Let $\left(\varphi_{U}\right)_{U \in \mathcal{U}}$ be a partition of unity on $X$ by $G$-invariant functions subordinate to this covering.

Since each $U \in \mathcal{U}$ comes with a map $f: U \rightarrow G / H$ for some finite subgroup $H$, we can decompose $U$ into a disjoint union $U=\bigsqcup_{g H \in G / H} f^{-1}(g H)$. We let $\mathcal{U}^{\prime}$ be the resulting open covering by these subsets. It still has the same finite covering dimension, so that its nerve $\left|\mathcal{U}^{\prime}\right|$ is a finite-dimensional simplicial complex. The obvious action of $G$ on $\mathcal{U}^{\prime}$ induces a proper simplicial action on $\left|\mathcal{U}^{\prime}\right|$. Let 
$\varphi_{f^{-1}(g H)}:=\left.\varphi_{U}\right|_{f^{-1}(g H)}$. This is a $G$-equivariant partition of unity on $X$. It induces a $G$-equivariant continuous map $X \rightarrow Y$. The assumption on $G$ ensures that the isotropy groups in $\left|\mathcal{U}^{\prime}\right|$ have finite order.

Thus [19, Corollary 2.7] provides a full $G$-equivariant vector bundle on $\left|\mathcal{U}^{\prime}\right|$, which pulls back to a full $G$-equivariant vector bundle on $X$. Now Theorem 6.14 yields a full projection and an approximate unit of projections in $\sigma-C^{*}(G \ltimes X)_{\mathbb{K}}$.

Finally, we reduce case (3) to case (2). There are full $G / K_{n}$-equivariant vector bundles on $K_{n} \backslash X$ for all $n \in \mathbb{N}$ by (2). Pull them back to $G$-equivariant vector bundles $V_{n}$ on $X$. For $x \in X$, any representation of the stabiliser $G_{x}^{x}$ must be trivial on $K_{n}$ for sufficiently large $n$ and hence be contained in the fibre of $V_{n}$. Hence the set of equivariant vector bundles $V_{n}$ is full.

If Theorem 6.15 applies and the action of $G$ on $X$ is cocompact, then Theorem 6.4 shows that $\mathrm{RK}_{G}^{0}(X)$ is generated by vector bundles.

Example 6.16. If a groupoid $\mathcal{G}$ acts freely on $X$, that is, all stabilisers are trivial, then the trivial 1-dimensional vector bundle with the obvious $\mathcal{G}$-action is full, so that $\sigma-C^{*}(\mathcal{G} \ltimes X)$ is Morita equivalent to a unital $\sigma-C^{*}$-algebra. If $\mathcal{G}$ already acts freely on its object space, that is, $\mathcal{G}$ describes an equivalence relation, then any action of $\mathcal{G}$ is free, so that the above applies to all proper actions of $\mathcal{G}$.

Example 6.17. Recall that orbifolds are described by effective proper étale Lie groupoids. [23, Theorem 4.1] asserts that any orbifold groupoid $\mathcal{G}$ is Morita equivalent to $L \ltimes X$ for an action of a compact group $L$ on a smooth manifold $X$. More precisely, we can let $X$ be the $\mathcal{G}$-orbit space of the frame bundle of $\mathcal{G}^{(0)}$ and $L$ be the orthogonal group of appropriate dimension.

Hence $\sigma-C^{*}(\mathcal{G})_{\mathbb{K}}$ contains an approximate unit of projections, so that the equivariant K-theory of a cocompact orbifold groupoid is generated by equivariant vector bundles.

\section{CONClusion}

We have defined K-theory groups with several support conditions that are equivariant with respect to proper actions of groupoids. While they are originally defined using bivariant $\mathrm{K}$-theory for $C^{*}$-algebras, their topological nature is made clear by alternative descriptions using homotopy classes of equivariant maps to suitable spaces of Fredholm operators. Another description using $\sigma$ - $C^{*}$-algebras is most convenient for establishing the formal properties of these theories.

A difficult question is when equivariant K-theory can be described by equivariant vector bundles or, more precisely, whether the Grothendieck group of the monoid of equivariant vector bundles on a space agrees with its equivariant representable K-theory. For cocompact actions, we have found a useful criterion that allows to study this question in examples, reducing it to the question: which stable $C^{*}$-algebras contain an approximate unit of projections?

New difficulties appear for actions that are not cocompact. Here we should assume finite covering dimension of the orbit space and the existence of a full equivariant vector bundle to achieve anything. So far, there appear to be no general results in this case that involve non-trivial groups. We plan to remedy this in a forthcoming article.

\section{REFERENCES}

[1] Herbert Abels, Parallelizability of proper actions, global $K$-slices and maximal compact subgroups, Math. Ann. 212 (1974/75), 1-19 MR 0375264

[2] $\quad$ A universal proper G-space, Math. Z. 159 (1978), no. 2, 143-158 MR 0501039 
[3] Michael Francis Atiyah, K-Theory, Lecture notes by D. W. Anderson, W. A. Benjamin, Inc., New York-Amsterdam, 1967 MR 0224083

[4] Bruce Blackadar, K-theory for operator algebras, 2nd ed., Mathematical Sciences Research Institute Publications, vol. 5, Cambridge University Press, Cambridge, 1998 MR 1656031

[5] Alexander Bonkat, Bivariante K-Theorie für Kategorien projektiver Systeme von $C^{*}$-Algebren, Ph.D. Thesis, Westf. Wilhelms-Universität Münster, 2002 (German). electronically available at the Deutsche Nationalbibliothek at http://deposit.ddb.de/cgi-bin/dokserv?idn=967387191

[6] Lawrence G. Brown, Stable isomorphism of hereditary subalgebras of $C^{*}$-algebras, Pacific J. Math. 71 (1977), no. 2, 335-348,MR 0454645

[7] Jérôme Chabert, Siegfried Echterhoff, and Ralf Meyer, Deux remarques sur l'application de Baum-Connes, C. R. Acad. Sci. Paris Sér. I Math. 332 (2001), no. 7, 607-610 (French) MR 1841893

[8] Joachim Cuntz and Nigel Higson, Kuiper's theorem for Hilbert modules, Operator algebras and mathematical physics (Iowa City, Iowa, 1985), Contemp. Math., vol. 62, Amer. Math. Soc., Providence, RI, 1987, pp. 429-435 MR 878392

[9] Joachim Cuntz, Ralf Meyer, and Jonathan M. Rosenberg, Topological and bivariant $K$-theory, Oberwolfach Seminars, vol. 36, Birkhäuser Verlag, Basel, 2007 MR 2340673

[10] Jacques Dixmier, $C^{*}$-Algebras, North-Holland Publishing Co., Amsterdam, 1977. Translated from the French by Francis Jellett; North-Holland Mathematical Library, Vol. 15 MR 0458185

[11] Siegfried Echterhoff, Heath Emerson, and Hyun Jeong Kim, KK-Theoretic duality for proper twisted actions (2006), eprint. arXiv: math/0610044

[12] Heath Emerson and Ralf Meyer, A descent principle for the Dirac-dual-Dirac method, Topology 46 (2007), no. 2, 185-209 MR 2313071

[13] _ Dualities in equivariant Kasparov theory (2007), eprint. arXiv: 0711.0025

[14] Mark A. Hennings, Kasparov's technical lemma for $b^{*}$-algebras, Math. Proc. Cambridge Philos. Soc. 105 (1989), no. 3, 537-545 MR 985690

[15] Klaus Jänich, Vektorraumbündel und der Raum der Fredholm-Operatoren, Math. Ann. 161 (1965), 129-142 MR 0190946

[16] Pierre Julg, K-Théorie équivariante et produits croisés, C. R. Acad. Sci. Paris Sér. I Math. 292 (1981), no. 13, 629-632 (French, with English summary) MR 625361

[17] Gennadi G. Kasparov, Equivariant KK-theory and the Novikov conjecture, Invent. Math. 91 (1988), no. 1, 147-201 MR 918241

[18] Gennadi G. Kasparov and Georges Skandalis, Groups acting properly on "bolic" spaces and the Novikov conjecture, Ann. of Math. (2) 158 (2003), no. 1, 165-206 MR 1998480

[19] Wolfgang Lück and Bob Oliver, The completion theorem in K-theory for proper actions of a discrete group, Topology 40 (2001), no. 3, 585-616 MR 1838997

[20] Albert T. Lundell and Stephen Weingram, The topology of $C W$ complexes, Van Nostrand Reinhold, New York, 1969.

[21] Pierre-Yves Le Gall, Théorie de Kasparov équivariante et groupoïdes. I, K-Theory 16 (1999), no. 4, 361-390 (French, with English and French summaries) MR 1686846

[22] James A. Mingo and William J. Phillips, Equivariant triviality theorems for Hilbert $C^{*}$-modules, Proc. Amer. Math. Soc. 91 (1984), no. 2, 225-230 MR 740176

[23] Ieke Moerdijk and Dorette A. Pronk, Orbifolds, sheaves and groupoids, K-Theory 12 (1997), no. 1, 3-21 MR 1466622

[24] Paul S. Muhly, Jean N. Renault, and Dana P. Williams, Equivalence and isomorphism for groupoid $C^{*}$-algebras, J. Operator Theory 17 (1987), no. 1, 3-22 MR 873460

[25] Alan L. T. Paterson, Groupoids, inverse semigroups, and their operator algebras, Progress in Mathematics, vol. 170, Birkhäuser Boston Inc., Boston, MA, 1999 MR 1724106

[26] N. Christopher Phillips, Inverse limits of $C^{*}$-algebras, J. Operator Theory 19 (1988), no. 1, 159-195.MR 950831

[27] _ Equivariant $K$-theory for proper actions. II. Some cases in which finite-dimensional bundles suffice, (New Orleans, LA/Indianapolis, IN, 1986), Contemp. Math., vol. 70, Amer. Math. Soc., Providence, RI, 1988, pp. 205-227 MR 948694

[28] Equivariant K-theory for proper actions, Pitman Research Notes in Mathematics Series, vol. 178, Longman Scientific \& Technical, Harlow, 1989 MR 991566

[29], Representable $K$-theory for $\sigma-C^{*}$-algebras, $K$-Theory 3 (1989), no. 5, 441478 MR 1050490

[30] Jean Renault, A groupoid approach to $\mathrm{C}^{*}$-algebras, Lecture Notes in Mathematics, vol. 793, Springer, Berlin, 1980 MR 584266

[31] L Représentation des produits croisés d'algèbres de groupoïdes, J. Operator Theory 18 (1987), no. 1, 67-97 (French) MR 912813 
[32] Juliane Sauer, K-Theory for proper smooth actions of totally disconnected groups, Highdimensional manifold topology, World Sci. Publ., River Edge, NJ, 2003, pp. 427448 MR 2048732

[33] Graeme Segal, Fredholm complexes, Quart. J. Math. Oxford Ser. (2) 21 (1970), 385402 MR 0271930

[34] Jean-Louis $\mathrm{Tu}$, La conjecture de Novikov pour les feuilletages hyperboliques, K-Theory 16 (1999), no. 2, 129-184 (French, with English and French summaries) MR 1671260

[35] Jean-Louis $\mathrm{Tu}$, Ping $\mathrm{Xu}$, and Camille Laurent-Gengoux, Twisted $K$-theory of differentiable stacks, Ann. Sci. École Norm. Sup. (4) 37 (2004), no. 6, 841-910 (English, with English and French summaries) MR 2119241

[36] Niels Erik Wegge-Olsen, K-Theory and $C^{*}$-algebras, Oxford Science Publications, The Clarendon Press Oxford University Press, New York, 1993 MR 1222415

[37] Joel J. Westman, Non-transitive groupoid algebras, Ph.D. Thesis, University of California at Irvine, 1967.

E-mail address: hemerson@math.uvic.ca

Department of Mathematics and Statistics, University of Victoria, PO BOX 3045 STN CSC, Victoria, B.C., Canada V8W 3P4

E-mail address: rameyer@uni-math.gwdg.de

Mathematisches Institut, Georg-August Universität Göttingen, Bunsenstrasse 3-5, 37073 Göttingen, GERMany 